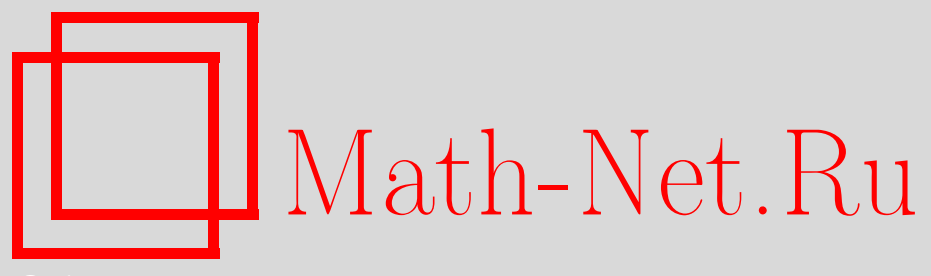

М. И. Вишик, В. В. Чепыжов, О траекторных аттракторах систем реакции-диффузии с малой диффузией, $M a-$ тем. сб., 2009, том 200, номер 4, 3-30

DOI: https://doi.org/10.4213/sm7298

Использование Общероссийского математического портала Math-Net.Ru подразумевает, что вы прочитали и согласны с пользовательским соглашением http://www . mathnet.ru/rus/agreement

Параметры загрузки:

IP : 54.81 .137 .203

26 апреля 2023 г., 04:07:07

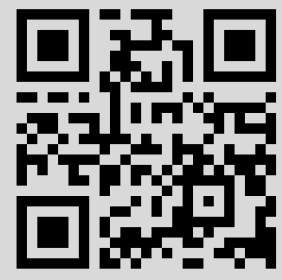




\author{
М. И. Вишик, В. В. Чепыжов
}

\title{
О траекторных аттракторах систем реакции-диффузии с малой диффузией
}

\begin{abstract}
Рассматривается система двух уравнений реакции-диффузии, из которых одно имеет малый коэффициент диффузии $\delta>0$. Построен траекторный аттрактор $\mathfrak{A}^{\delta}$ такой системы уравнений. Изучена предельная система уравнений при $\delta=0$. В этой системе одно из уравнений является обыкновенным дифференциальным уравнением по $t$, однако оно рассматривается в области $\Omega \times \mathbb{R}_{+}$, где $\Omega \Subset \mathbb{R}^{n}$ и $\mathbb{R}_{+}-$полуось времени $t$. Построен траекторный аттрактор $\mathfrak{A}^{0}$ предельной системы. Доказана основная теорема о сходимости: $\mathfrak{A}^{\delta} \rightarrow \mathfrak{A}^{0}$ при $\delta \rightarrow 0^{+}$в соответствующей топологии.

Библиография: 18 названий.
\end{abstract}

Ключевые слова: траекторный аттрактор, уравнения реакции-диффузии.

\section{Введение}

Системы уравнений реакции-диффузии и их глобальные аттракторы изучались во многих работах (см., например, книги [1]-[3] и цитированную в них литературу). При этом в основном рассматривались системы уравнений, для которых соответствующая задача Коши является корректно поставленной, т.е. по крайней мере она однозначно разрешима в подходящем функциональном пространстве, и, следовательно, ей соответствует динамическая полугруппа, действующая в фазовом пространстве начальных условий этой задачи. Кроме того, при соответствующих ограничениях из свойства сглаживания параболических уравнений следует существование у такой системы реакции-диффузии компактного поглощающего множества. Таким образом, соответствующая полугруппа является диссипативной и методами, изложенными в [1]-[5], можно построить глобальный аттрактор системы, а также доказать его конечномерность при выполнении некоторых дополнительных ограничений.

В настоящей статье исследуется модельная система реакции-диффузии, для которой не предполагается, что выполнена теорема единственности решения соответствующей задачи Коши. Тем не менее для этой системы можно строить глобальные слабые решения методом галёркинских приближений. Подобные системы часто встречаются в конкретных приложениях теории уравнений химической кинетики. Поэтому так важно уметь исследовать решения этих систем при больших временах. Однако методы теории глобальных аттракторов к таким уравнениям непосредственно не применимы. В настоящей статье подобные системы реакции-диффузии исследуются методом траекторных

Работа выполнена при финансовой поддержке Российского фонда фундаментальных исследований (гранты №№ 08-01-00784, 07-01-00500).

(C) М. И. Вишик, В. В. Чепыжов, 2009 
аттракторов, который весьма эффективен при исследовании слабых решений многих диссипативных уравнений математической физики, для которых не доказано, что соответствующая задача Коши является однозначно разрешимой (например, для трехмерной системы Навье-Стокса и других уравнений и систем; см., например, [3], [6]-[10]).

В ограниченной области $\Omega \Subset \mathbb{R}^{n}$ рассматриваются следующие уравнения:

$$
\begin{array}{ll}
\partial_{t} u=\Delta u-f(u, v)+g_{1}(x), & \left.u\right|_{\partial \Omega}=0, \\
\partial_{t} v=\delta \Delta v-h(u, v)+g_{2}(x), & \left.v\right|_{\partial \Omega}=0,
\end{array}
$$

где $\delta>0$ - малый параметр, а неизвестными являются скалярные функции $u=u(x, t)$ и $v=v(x, t), x \in \Omega, t \geqslant 0$. При выполнении соответствующих условий для нелинейных функций $f(u, v), h(u, v)$ и внешних сил $g_{1}(x), g_{2}(x)$ доказана теорема существования глобального решения в слабом смысле для системы уравнений (0.1)-(0.2). При этом для любого $M>0$

$$
\begin{aligned}
& u(\cdot) \in L_{p_{1}}\left(0, M ; L_{p_{1}}(\Omega)\right) \cap L_{2}\left(0, M ; H_{0}^{1}(\Omega)\right), \\
& v(\cdot) \in L_{p_{2}}\left(0, M ; L_{p_{2}}(\Omega)\right) \cap L_{2}\left(0, M ; H_{0}^{1}(\Omega)\right),
\end{aligned}
$$

где $\left(p_{1}, p_{2}\right)$ - показатели степенного роста по $(u, v)$ функций $(f, h)($ см. $\S 1)$. Кроме того, любое слабое решение $(u(x, t), v(x, t))$ системы $(0.1)-(0.2)$ удовлетворяет энергетическому тождеству, которое формально получается в результате скалярного умножения в $L_{2}(\Omega)$ уравнений $(0.1)$ и $(0.2)$ на $u(\cdot)$ и $v(\cdot)$ соответственно. Отсюда следует важное для дальнейшего энергетическое неравенство для суммы норм

$$
\|u(\cdot, t)\|_{L_{2}(\Omega)}^{2}+\|v(\cdot, t)\|_{L_{2}(\Omega)}^{2},
$$

а также для сумм интегралов

$$
\begin{gathered}
\int_{t}^{t+1}\left(\|u(\cdot, s)\|_{H_{0}^{1}(\Omega)}^{2}+\delta\|v(\cdot, s)\|_{H_{0}^{1}(\Omega)}^{2}\right) d s, \\
\int_{t}^{t+1}\left(\|u(\cdot, s)\|_{L_{p_{1}}(\Omega)}^{p_{1}}+\|v(\cdot, s)\|_{L_{p_{2}}(\Omega)}^{p_{2}}\right) d s
\end{gathered}
$$

при любом $t \geqslant 0$. Кроме этого в $\S 1$ для слабых решений, которые получаются в пределе из приближений Галёркина, доказывается равномерная (по $\delta>0$ ) оценка сверху для нормы

$$
\|v(\cdot, t)\|_{H_{0}^{1}(\Omega)}^{2}, \quad t \geqslant 0
$$

и, следовательно, для этих решений $v \in L_{\infty}\left(\mathbb{R}_{+} ; H_{0}^{1}(\Omega)\right)$. Отметим, что для слабого решения, которое нельзя аппроксимировать галёркинскими приближениями, получить эту оценку не удается.

На основании установленных неравенств для системы $(0.1)-(0.2)$ в $\S 2$ строится траекторный аттрактор. Для этого вводится банахово пространство $\mathscr{F}_{+}^{\mathrm{b}}$, состоящее из пар функций $(y(x, t), z(x, t)), t \geqslant 0$, обладающих равномерно ограниченными нормами $(0.5),(0.6)$ и $(0.7)$ при $t \geqslant 0$ (в этих формулах следует заменить $u(\cdot)$ и $v(\cdot)$ на $y(\cdot)$ и $z(\cdot))$. Кроме того, рассматривается более широкое 
пространство Фреше $\mathscr{F}_{+}^{\text {loc }} \supset \mathscr{F}_{+}^{\mathrm{b}}$, состоящее из указанных выше пар функций, для которых эти нормы ограничены на любом интервале $0 \leqslant t \leqslant M$. Пространство $\mathscr{F}_{+}^{\text {loc }}$ снабжается топологией $\Theta_{+}^{\text {loc }}$ слабой сходимости последовательностей функций $\left(y_{m}(\cdot, t), z_{m}(\cdot, t)\right)$ из $\mathscr{F}_{+}^{\text {loc } п р и ~} m \rightarrow \infty$ на каждом фиксированном интервале $[0, M]$.

Топология $\Theta_{+}^{\text {loc }}$ обладает следующим важным свойством: любой шар в пространстве $F_{+}^{\mathrm{b}}$ компактен в $\Theta_{+}^{\text {loc }}$.

Далее вводится (нелинейное) пространство траекторий $\mathscr{K}^{+}(N) \subset \mathscr{F}_{+}^{\text {loc }}, N \geqslant$ 0 , состоящее из всех слабых решений $(u(x, t), v(x, t))$ уравнений $(0.1)-(0.2)$, которые удовлетворяют неравенству

$$
\|v(t)\|_{H_{0}^{1}(\Omega)}^{2} \leqslant N \mathrm{e}^{-\sigma t}+R^{2}, \quad t \geqslant 0
$$

где $\sigma$ и $R$ совпадают с величинами, которые получаются при оценке норм $(0.7)$ соответствующих галёркинских приближений задачи (об этом см. подробнее в 1 ). Отметим, что $\sigma$ и $R$ не зависят от коэффициента диффузии $\delta$.

Устанавливается, что $\mathscr{K}^{+}(N) \subset \mathscr{F}_{+}^{\mathrm{b}}$ и $\mathscr{K}^{+}(N)$ замкнуто в топологии $\Theta_{+}^{\text {loc }}$. Отметим, что решения из $\mathscr{K}^{+}(N)$ обладают бо́льшей гладкостью, чем любые слабые решения системы (0.1)-(0.2).

Обозначим через $\{T(\tau), \tau \geqslant 0\}$ трансляционную полугруппу, которая действует на траекториях $(y(\cdot, t), z(\cdot, t)), t \geqslant 0$, по формуле

$$
T(\tau)(y(\cdot, t), z(\cdot, t))=(y(\cdot, t+\tau), z(\cdot, t+\tau)), \quad \tau \geqslant 0 .
$$

Устанавливается, что при любом $\tau \geqslant 0$ множество трансляций $T(\tau) \mathscr{K}^{+}(N)$ состоит из слабых решений уравнений $(0.1)-(0.2)$, для которых оценка $(0.8)$ также выполнена. Следовательно,

$$
T(\tau): \mathscr{K}^{+}(N) \rightarrow \mathscr{K}^{+}(N), \quad \tau \geqslant 0 .
$$

В $\S 2$ доказывается, что шар в $\mathscr{F}_{+}^{\mathrm{b}}$ достаточно большого радиуса служит поглощающим множеством полугруппы $\{T(\tau)\}$, действующей на $\mathscr{K}^{+}(N)$. Такой шар компактен в топологии $\Theta_{+}^{\text {loc }}$ и, следовательно, у трансляционной полугруппы имеется ограниченное в $\mathscr{F}_{+}^{\mathrm{b}}$ и компактное в $\Theta_{+}^{\mathrm{loc}}$ поглощающее множество. Отметим, что полугруппа $\{T(\tau)\}$ непрерывна в топологии $\Theta_{+}^{\text {loc }}$ на $\mathscr{K}^{+}(N)$. На основе этих свойств полугруппы $\{T(\tau)\}$ для нее строится траекторный аттрактор $\mathfrak{A} \subset \mathscr{K}_{+}(N)$, обладающий следующими свойствами:

1) множество $\mathfrak{A}$ ограничено в $\mathscr{F}_{+}^{\mathrm{b}}$ и компактно в топологии $\Theta_{+}^{\text {loc }}$;

2) $\mathfrak{A}$ строго инвариантно относительно полугруппы $\{T(\tau)\}$, т.е.

$$
T(\tau) \mathfrak{A}=\mathfrak{A}, \quad \tau \geqslant 0
$$

3) $\mathfrak{A}$ является притягивающим множеством $\{T(\tau)\}$ на $\mathscr{K}_{+}(N)$ в топологии $\Theta_{+}^{\text {loc }}$.

Доказывается, что $\mathfrak{A}$ не зависит от $N \geqslant 0$. Для удобства обозначим через $\mathfrak{A}^{\delta}$ построенный траекторный аттрактор системы (0.1)-(0.2), где $\delta$ - это малый коэффициент диффузии в уравнении (0.2). 
В $\S 3$ изучается система уравнений (0.1)-(0.2) при $\delta=0$. В этом случае уравнение (0.2) фактически становится обыкновенным дифференциальным уравнением относительно неизвестной функции $v(x, t), t \geqslant 0$, при каждом фиксированном $x \in \Omega$, причем на границе области $\partial \Omega$ задаются нулевые условия Дирихле: $v(x, t)=0, x \in \partial \Omega, t \geqslant 0$. Такие частично диссипативные системы реакции-диффузии рассматривались, например, в [11], где, однако, накладывались дополнительные ограничения на нелинейные члены уравнений, которые обеспечивали однозначную разрешимость соответствующей задачи Коши. Это свойство, как было отмечено выше, позволяет использовать теорию глобальных аттракторов при исследовании частично диссипативных систем реакции-диффузии, что было сделано в [11].

Оказывается, что частично диссипативные системы реакции-диффузии можно исследовать методами траекторных аттракторов, не требуя дополнительных ограничений, обеспечивающих единственность решения задачи Коши.

В $\S 3$ для случая $\delta=0$ строятся глобальные решения системы, удовлетворяющие оценкам для норм и интегралов $(0.5)-(0.7)$, аналогичных случаю $\delta>0$, рассмотренному в $\S 1$. Кроме того, эти решения удовлетворяют неравенству $(0.8)$ для подходящего $N \geqslant 0$. При каждом $N$ все такие решения, удовлетворяющие $(0.8)$, обозначаются через $\mathscr{K}_{+}^{0}(N)$. На пространстве $\mathscr{K}_{+}^{0}(N)$ рассматривается трансляционная полугруппа $\{T(\tau)\}$, причем пространство $\mathscr{K}_{+}^{0}(N)$ и полугруппа $\{T(\tau)\}$ имеют свойства, перечисленные выше для случая $\delta>0$.

$\mathrm{B} \S 3$ для частично диссипативной системы при $\delta=0$ находится траекторный аттрактор $\mathfrak{A}^{0}$ в топологии $\Theta_{+}^{\text {loc } п о л у г р у п п ы ~}\{T(\tau)\}$, действующей на пространстве траекторий $\mathscr{K}_{+}^{0}(N)$. Множество $\mathfrak{A}^{0}$ не зависит от $N$.

Частично диссипативную систему при $\delta=0$ можно назвать формальным "пределом" исходной системы реакции-диффузии $(0.1)-(0.2)$ при $\delta \rightarrow 0^{+}$. В $\S 4$ дается строгое обоснование предельному переходу, а именно, доказывается основная теорема настоящей статьи о сходимости траекторных аттракторов $\mathfrak{A}^{\delta}$ к $\mathfrak{A}^{0}$ в топологии $\Theta_{+}^{\text {loc } п р и ~} \delta \rightarrow 0^{+}$. Отметим, что в системе $(0.1)-(0.2)$ малый коэффициент $\delta>0$ стоит при старших производных по пространственным переменным, что свидетельствует о сильной сингулярности системы при переходе к пределу.

Приведенные выше результаты допускают обобщение на системы уравнений реакции-диффузии вида $(0.1)-(0.2)$, у которых неизвестными являются вектор-функции

$$
u=\left(u^{1}, u^{2}, \ldots, u^{k}\right), \quad v=\left(v^{k+1}, v^{k+2}, \ldots, v^{m}\right), \quad m \geqslant 2 .
$$

Таким системам будет посвящена отдельная работа авторов.

\section{§1. Уравнения реакции-диффузии, содержащие малый коэффициент диффузии $\delta>0$}

В ограниченной области $\Omega \Subset \mathbb{R}^{n}$ с границей $\partial \Omega \in C^{2}$ рассматривается система уравнений следующего вида:

$$
\begin{aligned}
& \partial_{t} u=\Delta u-f(u, v)+g_{1}(x), \\
& \partial_{t} v=\delta \Delta v-h(u, v)+g_{2}(x),
\end{aligned}
$$


в которой неизвестными являются скалярные функции $u=(x, t)$ и $v=v(x, t)$, $x \in \Omega, t \geqslant 0$. На границе $\partial \Omega$ задаются условия Дирихле

$$
\left.u\right|_{\partial \Omega}=0,\left.\quad v\right|_{\partial \Omega}=0 .
$$

В системе уравнений (1.1)-(1.2) оператор Лапласа $\Delta$ действует в области $\Omega$. В уравнении (1.2) коэффициент диффузии $\delta>0$ считается малым параметром. Коэффициент диффузии в уравнении (1.1) равен единице.

Предполагается, что нелинейные функции $f, h: \mathbb{R}^{2} \rightarrow \mathbb{R}$ являются непрерывными в $\mathbb{R}^{2}$ и удовлетворяют следующим условиям роста:

$$
\begin{gathered}
\sigma_{1}\left(|u|^{p_{1}}+|v|^{p_{2}}\right)-C \leqslant f(u, v) u+h(u, v) v \leqslant C_{0}\left(|u|^{p_{1}}+|v|^{p_{2}}+1\right), \\
|f(u, v)|^{q_{1}}+|h(u, v)|^{q_{2}} \leqslant C_{0}\left(|u|^{p_{1}}+|v|^{p_{2}}+1\right), \quad u, v \in \mathbb{R},
\end{gathered}
$$

где $\sigma_{1}, C, C_{0}, p_{1}, p_{2}$ - некоторые положительные константы, причем $p_{1}, p_{2} \geqslant 2$ и $q_{i}=p_{i} /\left(p_{i}-1\right), i=1,2$. Кроме того, предполагается, что

$$
h \in C^{1}\left(\mathbb{R}^{2}\right), \quad h(0,0)=0,
$$

и выполнены неравенства

$$
\begin{aligned}
\frac{\partial h}{\partial v}(u, v) & \geqslant \sigma_{2}>0, \\
\left|\frac{\partial h}{\partial u}(u, v)\right| & \leqslant D, \quad u, v \in \mathbb{R} .
\end{aligned}
$$

Отметим, что величины $\sigma_{1}>0$ и $\sigma_{2}>0$, ограничивающие снизу выражения в (1.4) и (1.7), отражают свойство диссипативности данной системы. Числа $\sigma_{i}$, $i=1,2$, могут быть произвольно малыми. Для определенности полагаем, что

$$
\sigma_{1}=\sigma_{2}=\sigma .
$$

Функции $g_{1}(x)$ и $g_{2}(x)$ в уравнениях (1.1) и (1.2) удовлетворяют условиям

$$
g_{1} \in L_{2}(\Omega), \quad g_{2} \in H_{0}^{1}(\Omega) .
$$

Для упрощения записи введем следующие обозначения:

$$
H:=L_{2}(\Omega), \quad V:=H_{0}^{1}(\Omega) .
$$

Норма в произвольном банаховом пространстве $X$ обозначается через $\|\cdot\|_{X}$, а нормы в пространствах $H$ и $V$ для краткости обозначаются через $\|\cdot\|$ и $\|\cdot\|_{1}$ соответственно. Напомним, что из неравенства Пуанкаре следует, что норму функции $w$ в пространстве $V=H_{0}^{1}(\Omega)$ можно задать по формуле

$$
\|w\|_{1}:=\|\nabla w\|=\left(\int_{\Omega} \sum_{i=1}^{n}\left|\partial_{x_{i}} w(x)\right|^{2} d x\right)^{1 / 2} .
$$

Отметим, что при выполнении перечисленных выше условий начальная задача Коши для системы (1.1)-(1.3) может иметь не одно решение в соответствующем пространстве. Это связано, в частности, с тем, что функция $f$ в уравнении (1.1) не предполагается дифференцируемой. 
Примером уравнений математической физики, для которой все указанные выше условия выполнены, служит модельная система Фитз-Хуг-Нагумо:

$$
\left\{\begin{array}{l}
\partial_{t} u=\Delta u-u(u-\beta)(u-1)-v, \\
\partial_{t} v=\delta \Delta v+\alpha u-\gamma v
\end{array}\right.
$$

где $f(u, v)=u(u-\beta)(u-1)+v, h(u, v)=\gamma v-\alpha u$ и коэффициенты $\alpha, \beta, \gamma$ являются положительными числами. Тогда $\sigma=\min \{1, \gamma\}$. В этом примере $p_{1}=4$, $p_{2}=2, g_{1} \equiv 0, g_{2} \equiv 0$. В приложениях обычно рассматриваются области $\Omega$ в пространстве размерности $n=1,2$ (см. [1], [12], [13]). Отметим, что задача Коши для системы (1.11) имеет единственное решение в соответствующих функциональных пространствах.

Для любых функций $u \in L_{p_{1}}\left(0, M ; L_{p_{1}}(\Omega)\right)$ и $v \in L_{p_{2}}\left(0, M ; L_{p_{2}}(\Omega)\right)$ из условия (1.5) следует, что

$$
f(u, v) \in L_{q_{1}}\left(0, M ; L_{q_{1}}(\Omega)\right), \quad h(u, v) \in L_{q_{2}}\left(0, M ; L_{q_{2}}(\Omega)\right),
$$

причем

$$
\begin{aligned}
& \|f(u, v)\|_{L_{q_{1}}\left(0, M ; L_{q_{1}}\right)}^{q_{1}}+\|h(u, v)\|_{L_{q_{2}}\left(0, M ; L_{q_{2}}\right)}^{q_{2}} \\
& \quad \leqslant C_{1}\left(\|u\|_{L_{p_{1}}\left(0, M ; L_{p_{1}}\right)}^{p_{1}}+\|v\|_{L_{p_{2}}\left(0, M ; L_{p_{2}}\right)}^{p_{2}}+1\right) .
\end{aligned}
$$

(Здесь и далее через $C_{i}$ обозначаются положительные константы, зависящие от функций $f, h$ и области $\Omega$, но не зависящие от параметра $\delta$.)

Если дополнительно известно, что $u, v \in L_{2}(0, M ; V)$, то

$$
\Delta u+g_{1}, \delta \Delta v+g_{2} \in L_{2}\left(0, M ; H^{-1}(\Omega)\right) .
$$

Из теоремы вложения Соболева следует, что $H_{0}^{r_{i}}(\Omega) \subset L_{p_{i}}(\Omega)$ при $r_{i} \geqslant n(1 / 2-$ $\left.1 / p_{i}\right)$, и тогда для сопряженных пространств имеем вложение

$$
L_{q_{i}}(\Omega) \subset H^{-r_{i}}(\Omega) .
$$

Поэтому если $r_{i} \geqslant 1$, то в силу (1.12) и (1.14) правые части уравнений (1.1) и (1.2) принадлежат пространствам $L_{q_{1}}\left(0, M ; H^{-r_{1}}(\Omega)\right)$ и $L_{q_{2}}\left(0, M ; H^{-r_{2}}(\Omega)\right)$ соответственно. Обозначим

$$
r_{i}=\max \left\{1, n\left(\frac{1}{2}-\frac{1}{p_{i}}\right)\right\}, \quad i=1,2 .
$$

Тогда решения для уравнений (1.1) и (1.2) можно искать в пространствах обобщенных функций $\mathscr{D}^{\prime}\left(0, M ; H^{-r_{1}}(\Omega)\right)$ и $\mathscr{D}^{\prime}\left(0, M ; H^{-r_{2}}(\Omega)\right)$ соответственно (см. [14]), причем

$$
\partial_{t} u \in L_{q_{1}}\left(0, M ; H^{-r_{1}}(\Omega)\right), \quad \partial_{t} v \in L_{q_{2}}\left(0, M ; H^{-r_{2}}(\Omega)\right) .
$$

Слабым решением системы (1.1)-(1.2) называется пара функций $(u(x, t)$, $v(x, t))$ такая, что в области $\Omega \times \mathbb{R}_{+}$при любом $M>0$

$$
\begin{aligned}
& u \in L_{p_{1}}\left(0, M ; L_{p_{1}}(\Omega)\right) \cap L_{2}(0, M ; V), \\
& v \in L_{p_{2}}\left(0, M ; L_{p_{2}}(\Omega)\right) \cap L_{2}(0, M ; V),
\end{aligned}
$$


причем $u(x, t)$ и $v(x, t)$ удовлетворяют уравнениям (1.1)-(1.2) в смысле теории обобщенных функций в $\mathscr{D}^{\prime}\left(0, M ; H^{-r_{1}}(\Omega) \times H^{-r_{2}}(\Omega)\right)($ см. [2], [3], [14]).

Из уравнений (1.1)-(1.3) находим: $u(\cdot) \in L_{\infty}(0, M ; H), v(\cdot) \in L_{\infty}(0, M ; H)$, и из известной леммы Лионса-Мадженеса [15] получаем, что

$$
u(\cdot) \in C_{w}([0, M] ; H), \quad v(\cdot) \in C_{w}([0, M] ; H), \quad M>0 .
$$

Следовательно, при каждом $t \geqslant 0$ определены значения $u(t), v(t)$ и, в частности, имеют смысл начальные условия вида

$$
\left.u\right|_{t=0}=u_{0} \in H,\left.\quad v\right|_{t=0}=v_{0} \in H
$$

Далее для краткости мы будем часто опускать пространственную переменную $x$ в аргументах функций $u$ и $v$.

Существование слабого решения системы (1.1)-(1.2) с начальными условиями (1.17) при любых $u_{0} \in H, v_{0} \in H$ устанавливается методом Галёркина, причем в качестве базисных функций выбираются собственные функции оператора Лапласа (см., например, [2], [3]). При этом стандартным образом доказываются энергетические оценки для галёркинских приближений $\left(u_{m}(t), v_{m}(t)\right)$ порядка $m \in \mathbb{N}$, из которых выбирается подпоследовательность с индексами $\left\{m^{\prime}\right\} \subset\{m\}$, сходящаяся при $m^{\prime} \rightarrow \infty$ в смысле пространства $\mathscr{D}^{\prime}\left(0, M ; H^{-r_{1}}(\Omega) \times H^{-r_{2}}(\Omega)\right)$ при любом $M>0$ к некоторому слабому решению $(u(t), v(t))$ задачи (1.1)-(1.2), (1.17) (см. [2], [3]).

Упомянутые выше энергетические оценки галёркинских приближений будут получены далее в виде априорных оценок для слабых решений системы (1.1)-(1.2). Строгое обоснование этих неравенств приведено в [3].

В [3] также доказано, что любое слабое решение $(u(\cdot), v(\cdot))$ системы вида (1.1)-(1.2) обладает следующими свойствами:

(i) $u(\cdot) \in C\left(\mathbb{R}_{+} ; H\right), v(\cdot) \in C\left(\mathbb{R}_{+} ; H\right)$;

(ii) вещественная функция $\|u(t)\|^{2}+\|v(t)\|^{2}$ является абсолютно непрерывной по $t \geqslant 0$ и удовлетворяет энергетическому тождеству

$$
\begin{aligned}
\frac{1}{2} \frac{d}{d t}\{ & \left.\|u(t)\|^{2}+\|v(t)\|^{2}\right\}+\|\nabla u(t)\|^{2}+\delta\|\nabla v(t)\|^{2} \\
& \quad+\int_{\Omega} f(u(x, t), v(x, t)) u(x, t) d x+\int_{\Omega} h(u(x, t), v(x, t)) v(x, t) d x \\
& =\left\langle g_{1}, u(t)\right\rangle+\left\langle g_{2}, v(t)\right\rangle .
\end{aligned}
$$

Здесь и далее символ $\langle\cdot, \cdot\rangle$ обозначает скалярное произведение функций в $H$. Тождество (1.18) формально получается скалярным умножением в $H$ уравнения (1.1) на $u(t)$, уравнения (1.2) - на $v(t)$, последующим сложением результатов и применением формул

$$
\frac{d}{d t}\|u(t)\|^{2}=2\left\langle\partial_{t} u(t), u(t)\right\rangle, \quad \frac{d}{d t}\|v(t)\|^{2}=2\left\langle\partial_{t} v(t), v(t)\right\rangle .
$$

Строгое обоснование этих формул для слабых решений системы (1.1)-(1.2) можно найти в [3]. 
Воспользовавшись неравенством (1.4), выводим из тождества (1.18) следующее дифференциальное неравенство:

$$
\begin{gathered}
\frac{1}{2} \frac{d}{d t}\left\{\|u(t)\|^{2}+\|v(t)\|^{2}\right\}+\left\{\|\nabla u(t)\|^{2}+\delta\|\nabla v(t)\|^{2}\right\} \\
\quad+\sigma \int_{\Omega}\left\{|u(x, t)|^{p_{1}}+|v(x, t)|^{p_{2}}\right\} d x \\
\leqslant\|u(t)\|\left\|g_{1}\right\|+\|v(t)\|\left\|g_{2}\right\|+C|\Omega|, \quad t \geqslant 0 .
\end{gathered}
$$

Из элементарного неравенства $|a|^{p} \geqslant|a|^{2}-1$ при $p \geqslant 2$ получаем из (1.19)

$$
\begin{gathered}
\frac{1}{2} \frac{d}{d t}\left\{\|u(t)\|^{2}+\|v(t)\|^{2}\right\}+\left\{\|\nabla u(t)\|^{2}+\delta\|\nabla v(t)\|^{2}\right\}+\sigma\left\{\|u(t)\|^{2}+\|v(t)\|^{2}\right\} \\
\leqslant\|u(t)\|\left\|g_{1}\right\|+\|v(t)\|\left\|g_{2}\right\|+C|\Omega|+2 \sigma .
\end{gathered}
$$

Применяя к правой части (1.20) неравенство Коши, находим

$$
\begin{gathered}
\frac{d}{d t}\left\{\|u(t)\|^{2}+\|v(t)\|^{2}\right\}+2\left\{\|\nabla u(t)\|^{2}+\delta\|\nabla v(t)\|^{2}\right\}+\sigma\left\{\|u(t)\|^{2}+\|v(t)\|^{2}\right\} \\
\leqslant \sigma^{-1}\left\|g_{1}\right\|^{2}+\sigma^{-1}\left\|g_{2}\right\|^{2}+2 C|\Omega|+4 \sigma .
\end{gathered}
$$

Обозначив для краткости

$$
\begin{gathered}
\zeta(t)=\|u(t)\|^{2}+\|v(t)\|^{2}, \quad \phi(t)=2\left\{\|\nabla u(t)\|^{2}+\delta\|\nabla v(t)\|^{2}\right\}, \\
R_{0}^{2}=\sigma^{-1}\left\|g_{1}\right\|^{2}+\sigma^{-1}\left\|g_{2}\right\|^{2}+2 C|\Omega|+4 \sigma
\end{gathered}
$$

получим неравенство

$$
\frac{d}{d t} \zeta(t)+\phi(t)+\sigma \zeta(t) \leqslant R_{0}^{2}
$$

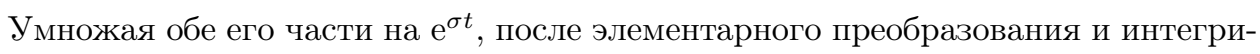
рования по времени приходим к следующим неравенствам:

$$
\begin{gathered}
\frac{d}{d t}\left[\zeta(t) \mathrm{e}^{\sigma t}\right]+\phi(t) \mathrm{e}^{\sigma t} \leqslant R_{0}^{2} \mathrm{e}^{\sigma t} \\
\zeta(t) \mathrm{e}^{\sigma t}-\zeta(0)+\int_{0}^{t} \phi(s) \mathrm{e}^{\sigma s} d s \leqslant R_{0}^{2} \sigma^{-1}\left[\mathrm{e}^{\sigma t}-1\right] \leqslant R_{0}^{2} \sigma^{-1} \mathrm{e}^{\sigma t} .
\end{gathered}
$$

Подставив выражения для $\zeta$ и $\phi$, получаем оценку

$$
\begin{gathered}
\|u(t)\|^{2}+\|v(t)\|^{2}+2 \int_{0}^{t}\left\{\|\nabla u(s)\|^{2}+\delta\|\nabla v(s)\|^{2}\right\} \mathrm{e}^{-\sigma(t-s)} d s \\
\leqslant\left(\|u(0)\|^{2}+\|v(0)\|^{2}\right) \mathrm{e}^{-\sigma t}+R_{1}^{2},
\end{gathered}
$$

где

$$
R_{1}^{2}:=R_{0}^{2} \sigma^{-1}=\sigma^{-2}\left\|g_{1}\right\|^{2}+\sigma^{-2}\left\|g_{2}\right\|^{2}+2 \sigma^{-1} C|\Omega|+4 .
$$

Заметим, что величина $R_{1}$ не зависит от $\delta$. 
Из тождества (1.18), рассуждая как и при выводе (1.19)-(1.21) и применяя неравенство Юнга, получаем неравенство

$$
\begin{gathered}
\frac{d}{d t}\left\{\|u(t)\|^{2}+\|v(t)\|^{2}\right\}+2\|\nabla u(t)\|^{2}+2 \delta\|\nabla v(t)\|^{2} \\
+\sigma \int_{\Omega}\left\{|u(x, t)|^{p_{1}}+|u(x, t)|^{p_{2}}\right\} d x \\
\leqslant C_{2}\left(\left\|g_{1}\right\|^{2}+\left\|g_{2}\right\|^{2}+1\right), \quad t \geqslant 0,
\end{gathered}
$$

где константа $C_{2}$ зависит от $\sigma$ и не зависит от $g_{1}$ и $g_{2}$. Интегрируя неравенство $(1.24)$ на отрезке $[t, t+1]$, приходим к неравенству

$$
\begin{gathered}
\left(\|u(t+1)\|^{2}+\|v(t+1)\|^{2}\right)+2 \int_{t}^{t+1}\left(\|u(s)\|_{1}^{2}+\delta\|v(s)\|_{1}^{2}\right) d s \\
+\sigma \int_{t}^{t+1}\left(\|u(s)\|_{L_{p_{1}}}^{p_{1}}+\|v(s)\|_{L_{p_{2}}}^{p_{2}}\right) d s \\
\leqslant\left(\|u(t)\|^{2}+\|v(t)\|^{2}\right)+C_{2}\left(\left\|g_{1}\right\|^{2}+\left\|g_{2}\right\|^{2}+1\right),
\end{gathered}
$$

откуда с учетом (1.22) следует, что

$$
\begin{aligned}
2 \int_{t}^{t+1} & \left(\|u(s)\|_{V}^{2}+\delta\|v(s)\|_{V}^{2}\right) d s+\sigma \int_{t}^{t+1}\left(\|u(s)\|_{L_{p_{1}}}^{p_{1}}+\|v(s)\|_{L_{p_{2}}}^{p_{2}}\right) d s \\
\leqslant & \left(\|u(0)\|^{2}+\|v(0)\|^{2}\right) \mathrm{e}^{-\sigma t}+R_{2}^{2},
\end{aligned}
$$

где

$$
R_{2}^{2}:=R_{1}^{2}+C_{2}\left(\left\|g_{1}\right\|^{2}+\left\|g_{2}\right\|^{2}+1\right) .
$$

Таким образом, мы доказали следующее

УТВЕРЖДЕНИЕ 1.1. Следующие неравенства справедливы для любого слабого решения $(u(t), v(t))$ задачи (1.1)-(1.2), (1.17):

$$
\begin{gathered}
\|u(t)\|^{2}+\|v(t)\|^{2}+2 \int_{0}^{t}\left(\|\nabla u(s)\|^{2}+\delta\|\nabla v(s)\|^{2}\right) \mathrm{e}^{-\sigma(t-s)} d s \\
\leqslant\left(\left\|u_{0}\right\|^{2}+\left\|v_{0}\right\|^{2}\right) \mathrm{e}^{-\sigma t}+R_{1}^{2}, \\
2 \int_{t}^{t+1}\left(\|u(s)\|_{V}^{2}+\delta\|v(s)\|_{V}^{2}\right) d s+\sigma \int_{t}^{t+1}\left(\|u(s)\|_{L_{p_{1}}}^{p_{1}}+\|v(s)\|_{L_{p_{2}}}^{p_{2}}\right) d s \\
\leqslant\left(\left\|u_{0}\right\|^{2}+\left\|v_{0}\right\|^{2}\right) \mathrm{e}^{-\sigma t}+R_{2}^{2}, \quad t \geqslant 0 .
\end{gathered}
$$

Установим еще одну важную априорную оценку для нормы в пространстве $V$ компоненты $v$ слабого решения $(u(t), v(t))$ задачи $(1.1)-(1.2),(1.17)$, которое получается по методу Галёркина в пределе при $m \rightarrow \infty$ из соответствующих приближений $\left(u_{m}(t), v_{m}(t)\right)$ при условии, что

$$
\left.v\right|_{t=0}=v_{0} \in V
$$

ЗАмечАниЕ 1.1. Отметим, что любое слабое решение задачи (1.1)-(1.2), (1.17) из класса функций (1.15)-(1.16) не обязательно будет удовлетворять этой 
дополнительной априорной оценке. Однако именно эта оценка позволит нам в дальнейшем исследовать систему реакции-диффузии при (1.1)-(1.2) для предельного случая $\delta=0$. Метод Галёркина поможет обнаружить слабые решения, для которых эта специальная оценка имеет место при условии, что начальные данные удовлетворяют (1.29), что сильнее предположения (1.17).

Умножим уравнение (1.2) на $-\Delta v$ и получим после интегрирования по переменной $x$ в области $\Omega$ следующее тождество:

$$
\frac{1}{2} \frac{d}{d t}\|v(t)\|_{1}^{2}+\delta\|\Delta v(t)\|^{2}-\int_{\Omega} h(u(x, t), v(x, t)) \Delta v(x, t) d x=\left\langle\nabla g_{2}, \nabla v(t)\right\rangle .
$$

Здесь мы воспользовались равенствами

$$
-\left\langle\partial_{t} v, \Delta v\right\rangle=\frac{1}{2} \frac{d}{d t}\|v(t)\|_{1}^{2}, \quad-\left\langle g_{2}, \Delta v\right\rangle=\left\langle\nabla g_{2}, \nabla v(t)\right\rangle
$$

которые справедливы для достаточно гладких функций $v(x, t)$, т.е. для таких, для которых

$$
v \in L_{2}\left(0, M ; H_{0}^{1}(\Omega) \cap H^{2}(\Omega)\right), \quad \partial_{t} v \in L_{2}\left(0, M ; L_{2}(\Omega)\right)
$$

(см., например, [3]). Эти включения выполнены для любого галёркинского приближения $v_{m}(t)$ при условии (1.29). Подчеркнем, что произвольное слабое решение системы (1.1)-(1.2), (1.17), вообще говоря, не обязано удовлетворять тождеству (1.30). Это замечание также относится и к неравенству (1.31).

Продолжим вывод априорной оценки. Применяя интегрирование по частям в интеграле в левой части (1.30), содержащем функцию $h$, учитывая условие $h(0,0)=0$, а также используя нулевые граничные условия $(1.3)$, получим

$$
\begin{aligned}
& -\int_{\Omega} h(u, v) \Delta v d x=\int_{\Omega} \sum_{i=1}^{n} \partial_{x_{i}} h(u, v) \partial_{x_{i}} v d x \\
& \quad=\int_{\Omega} \sum_{i=1}^{n} \partial_{v} h(u, v)\left(\partial_{x_{i}} v\right)^{2} d x+\int_{\Omega} \sum_{i=1}^{n} \partial_{u} h(u, v) \partial_{x_{i}} u \partial_{x_{i}} v d x \\
& \geqslant \sigma\|v\|_{1}^{2}-D\|v\|_{1}\|u\|_{1} .
\end{aligned}
$$

Здесь мы воспользовались условиями (1.7)-(1.8). Применяя неравенство Коши, находим, что

$$
\begin{gathered}
D\|v\|_{1}\|u\|_{1} \leqslant \frac{1}{4} \sigma\|v\|_{1}^{2}+\sigma^{-1} D^{2}\|u\|_{1}^{2}, \\
\left\langle\nabla g_{2}, \nabla v\right\rangle \leqslant \frac{1}{4} \sigma\|v\|_{1}^{2}+\sigma^{-1}\left\|g_{2}\right\|_{1}^{2} .
\end{gathered}
$$

Тогда из (1.30) и неравенств (1.31)-(1.33) находим

$$
\frac{d}{d t}\left(\|v(t)\|_{1}^{2}\right)+2 \delta\|\Delta v(t)\|^{2}+\sigma\|v(t)\|_{1}^{2} \leqslant C_{3}\|u\|_{1}^{2}+C_{4}\left\|g_{2}\right\|_{1}^{2} .
$$

Пренебрегая членом с малым коэффициентом диффузии $\delta$, получаем

$$
\frac{d}{d t}\left(\|v(t)\|_{1}^{2}\right)+\sigma\|v(t)\|_{1}^{2} \leqslant C_{3}\|u\|_{1}^{2}+C_{4}\left\|g_{2}\right\|_{1}^{2}
$$


где

$$
C_{3}=2 \sigma^{-1} D^{2}, \quad C_{4}=2 \sigma^{-1} .
$$

Из (1.35) находим, что

$$
\frac{d}{d t}\left(\|v(t)\|_{1}^{2} \mathrm{e}^{\sigma t}\right) \leqslant C_{3}\|u\|_{1}^{2} \mathrm{e}^{\sigma t}+C_{4}\left\|g_{2}\right\|_{1}^{2} \mathrm{e}^{\sigma t} .
$$

Интегрируя по времени, имеем

$$
\begin{array}{r}
\|v(t)\|_{1}^{2} \mathrm{e}^{\sigma t} \leqslant\|v(0)\|_{1}^{2}+C_{3} \int_{0}^{t}\|u(s)\|_{1}^{2} \mathrm{e}^{\sigma s} d s+C_{4} \sigma^{-1}\left\|g_{2}\right\|_{1}^{2} \mathrm{e}^{\sigma t}, \\
\|v(t)\|_{1}^{2} \leqslant\|v(0)\|_{1}^{2} \mathrm{e}^{-\sigma t}+2 \sigma^{-1} D^{2} \int_{0}^{t}\|u(s)\|_{1}^{2} \mathrm{e}^{-\sigma(t-s)} d s+2 \sigma^{-2}\left\|g_{2}\right\|_{1}^{2} .
\end{array}
$$

Интеграл в правой части неравенства (1.36) оценивается с помощью неравенства (1.27) так:

$$
\int_{0}^{t}\|u(s)\|_{1}^{2} \mathrm{e}^{-\sigma(t-s)} d s \leqslant \frac{1}{2}\left(\|u(0)\|^{2}+\|v(0)\|^{2}\right) \mathrm{e}^{-\sigma t}+\frac{R_{1}^{2}}{2} .
$$

В итоге из (1.36) получаем оценку

$$
\|v(t)\|_{1}^{2} \leqslant\|v(0)\|_{1}^{2} \mathrm{e}^{-\sigma t}+C_{5}\left(\|u(0)\|^{2}+\|v(0)\|^{2}\right) \mathrm{e}^{-\sigma t}+R^{2},
$$

где

$$
R^{2}=\sigma^{-1} D^{2} R_{1}^{2}+2 \sigma^{-2}\left\|g_{2}\right\|_{1}^{2}, \quad C_{5}=\sigma^{-1} D^{2} .
$$

(Величина $R_{1}$ определена в (1.23).) Таким образом, доказано следующее

УтВЕРЖДЕНИЕ 1.2. Если выполнено условие (1.29), то задача (1.1)-(1.2), (1.17) имеет слабое решение $(u(t), v(t))$, для которого $v \in L_{\infty}\left(\mathbb{R}_{+} ; V\right)$, причем выполнено неравенство

$$
\|v(t)\|_{1}^{2} \leqslant\left\|v_{0}\right\|_{1}^{2} \mathrm{e}^{-\sigma t}+C_{5}\left(\left\|u_{0}\right\|^{2}+\left\|v_{0}\right\|^{2}\right) \mathrm{e}^{-\sigma t}+R^{2}, \quad t \geqslant 0,
$$

где величина $R$ и константа $C_{5}$ определенъ в (1.37) и они не зависят от $\delta$.

Из упомянутой ранее леммы Лионса-Мадженеса следует, что найденное выше слабое решение $v \in C_{w}\left(\mathbb{R}_{+} ; V\right)$, т.е. при каждом $t \geqslant 0$ корректно определены значения $v(t) \in V$ и неравенство (1.38) выполнено при любом $t \geqslant 0$.

ЗАмЕчАниЕ 1.2. Построенное в утверждении 1.2 слабое решение $(u(t), v(t))$ задачи (1.1)-(1.2), (1.17) обладает также дополнительной гладкостью, т.е.

$$
v \in L_{2}\left(0, M ; H^{2}(\Omega)\right), \quad M>0 .
$$

Чтобы в этом убедиться, достаточно сохранить в неравенстве (1.34) член $2 \delta\|\Delta v(t)\|^{2}$ и совершить последующие преобразования неравенств. Тогда в итоговых оценках (1.37) и (1.38) слева останется еще одно слагаемое

$$
2 \delta \int_{0}^{t}\|\Delta v(s)\|^{2} \mathrm{e}^{-\sigma(t-s)} d s
$$


которое позволит оценить сверху интеграл

$$
2 \delta \int_{t}^{t+1}\|\Delta v(s)\|^{2} d s \quad t \geqslant 0
$$

Однако оценки сверху для нормы $\int_{t}^{t+1}\|\Delta v(s)\|^{2} d s$, получаемые после этого из (1.38), не будут равномерными, когда $\delta$ близко к нулю. Поэтому свойство гладкости (1.39) не сохранится при $\delta \rightarrow 0^{+}$.

\section{§ 2. Траекторный аттрактор системы реакции-диффузии}

Введем пространства $\mathscr{F}_{+}^{\text {loc }}, \mathscr{F}_{+}^{\mathrm{b}}$ и топологию $\Theta_{+}^{\text {loc }}$ слабой секвенциальной сходимости в пространстве $\mathscr{F}_{+}^{\text {loc }}$. По определению

$$
\mathscr{F}_{+}^{\text {loc }}=\left\{\begin{array}{c}
(y(x, t), z(x, t)), x \in \Omega, t \geqslant 0 \mid \\
y \in L_{\infty}^{\mathrm{loc}}\left(\mathbb{R}_{+} ; H\right) \cap L_{2}^{\mathrm{loc}}\left(\mathbb{R}_{+} ; V\right) \cap L_{p_{1}}^{\text {loc }}\left(\mathbb{R}_{+} ; L_{p_{1}}(\Omega)\right), \\
z \in L_{\infty}^{\mathrm{loc}}\left(\mathbb{R}_{+} ; V\right) \cap L_{p_{2}}^{\mathrm{loc}}\left(\mathbb{R}_{+} ; L_{p_{2}}(\Omega)\right), \\
\partial_{t} y \in L_{q_{1}}^{\mathrm{loc}}\left(\mathbb{R}_{+} ; H^{-r_{1}}(\Omega)\right), \partial_{t} z \in L_{q_{2}}^{\text {loc }}\left(\mathbb{R}_{+} ; H^{-r_{2}}(\Omega)\right)
\end{array}\right\}
$$

В пространстве $\mathscr{F}_{+}^{\text {loc }}$ рассматривается следующая топология, которая для простоты определяется с помощью задания сходимости соответствующих последовательностей. Согласно определению последовательность функций $\left\{\left(y_{m}(\cdot), z_{m}(\cdot)\right)\right\} \subset \mathscr{F}_{+}^{\text {loc }}$ сходится при $m \rightarrow \infty$ к некоторой функции $(y(\cdot), z(\cdot)) \in \mathscr{F}_{+}^{\text {loc }}$ в топологии $\Theta_{+}^{\text {loc }}$, если при $m \rightarrow \infty$ при любом $M>0$ имеют место следующие сходимости:

1) $y_{m}(\cdot) \rightarrow y(\cdot) *$-слабо в $L_{\infty}(0, M ; H)$, слабо в $L_{2}(0, M ; V)$ и слабо в $L_{p_{1}}\left(0, M ; L_{p_{1}}(\Omega)\right)$;

2) $z_{m}(\cdot) \rightarrow z(\cdot) *$-слабо в $L_{\infty}(0, M ; V)$ и слабо в $L_{p_{2}}\left(0, M ; L_{p_{2}}(\Omega)\right)$;

3) $\partial_{t} y_{m}(\cdot) \rightarrow \partial_{t} y(\cdot)$ слабо в $L_{q_{1}}\left(0, M ; H^{-r_{1}}(\Omega)\right)$;

4) $\partial_{t} z_{m}(\cdot) \rightarrow \partial_{t} z(\cdot)$ слабо в $L_{q_{2}}\left(0, M ; H^{-r_{2}}(\Omega)\right)$.

Пространство $\mathscr{F}_{+}^{\text {loc }}$ с топологией $\Theta_{+}^{\text {loc }}$ является линейным хаусдорфовым пространством Фреше-Урысона со счетной базой (см. [3]). В нем рассматривается линейное подпространство $\mathscr{F}_{+}^{\mathrm{b}} \subset \mathscr{F}_{+}^{\text {loc }}$, состоящее из функций $(y, z)$ с конечной нормой

$$
\begin{gathered}
\|(y, z)\|_{\mathscr{F}_{+}^{\mathrm{b}}}:=\|y\|_{L_{\infty}\left(\mathbb{R}_{+} ; H\right)}+\|y\|_{L_{2}^{\mathrm{b}}\left(\mathbb{R}_{+} ; V\right)}+\|y\|_{L_{p_{1}}^{\mathrm{b}}\left(\mathbb{R}_{+} ; L_{p_{1}}\right)}+\left\|\partial_{t} y\right\|_{L_{q_{1}}^{\mathrm{b}}\left(\mathbb{R}_{+} ; H^{-r_{1}}\right)} \\
+\|z\|_{L_{\infty}\left(\mathbb{R}_{+} ; V\right)}+\|z\|_{L_{p_{2}}^{\mathrm{b}}\left(\mathbb{R}_{+} ; L_{p_{2}}\right)}+\left\|\partial_{t} z\right\|_{L_{q_{2}}^{\mathrm{b}}\left(\mathbb{R}_{+} ; H^{-r_{2}}\right)} .
\end{gathered}
$$

Напомним, что норма в пространстве $L_{p}^{\mathrm{b}}\left(\mathbb{R}_{+} ; X\right), p \geqslant 1, X$ - банахово пространство, задается по формуле

$$
\|y\|_{L_{p}^{\mathrm{b}\left(\mathbb{R}_{+} ; X\right)}}^{p}:=\sup _{t \geqslant 0} \int_{t}^{t+1}\|y(s)\|_{X}^{p} d s .
$$

Легко видеть, что пространство $\mathscr{F}_{+}^{\mathrm{b}}$ с нормой $(2.2)$ является пространством Банаха. 
ЗАМЕЧАНИЕ 2.1. Любой шар

$$
B_{r}=\left\{\|(y, z)\|_{\mathscr{F}_{+}^{\mathrm{b}}} \leqslant r\right\}
$$

в пространстве $\mathscr{F}_{+}^{\mathrm{b}}$ является компактным множеством в топологии $\Theta_{+}^{\text {loc }}$. Это следует из свойства слабой и $*$-слабой компактности ограниченных множеств в соответствующих банаховых пространствах (см., например, [3]). Тогда из общих теорем о компактных топологических пространств следует, что множество $B_{r}$ с топологией, индуцированной из $\Theta_{+}^{\text {loc }}$, является метризуемым пространством (см., например, [16]). Это свойство будет использоваться при построении траекторного аттрактора изучаемой системы. (Отметим, что пространство $\mathscr{F}_{+}^{\text {loc }}$ с топологией $\Theta_{+}^{\text {loc }}$ не является метризуемым.)

Определим теперь пространство $\mathscr{K}_{+}(N)$ решений (траекторий) системы (1.1)-(1.2), которое зависит от числа $N>0$.

ОПРЕДЕЛЕНИЕ 2.1. Пространство $\mathscr{K}_{+}(N)$ состоит из функций $(u(\cdot), v(\cdot)) \in$ $\mathscr{F}_{+}^{\text {loc }}$ таких, что

(i) пара функций $(u(t), v(t)), t \geqslant 0$, является слабым решением системы $(1.1)-(1.2)$

(ii) функция $v(t)$ удовлетворят неравенству

$$
\|v(t)\|_{1}^{2} \leqslant N \mathrm{e}^{-\sigma t}+R^{2}, \quad t \geqslant 0
$$

где величины $\sigma$ и $R$ те же, что и в неравенстве (1.38).

Напомним, что если $(u(\cdot), v(\cdot)) \in \mathscr{F}_{+}^{\text {loc }}$ и $(u(t), v(t))$ является слабым решением системы (1.1)-(1.2), то в силу леммы Лионса-Мадженеса $v \in C_{w}\left(\mathbb{R}_{+} ; V\right)$ и неравенство (2.3) имеет смысл при всех $t \geqslant 0$.

Заметим, что траекторное пространство $\mathscr{K}_{+}(N)$ не пусто. Действительно, решая методом Галёркина задачу Коши (1.1)-(1.2), (1.17) при $u_{0} \in H, v_{0} \in V$, мы получим слабое решение $(u(t), v(t))$ этой системы, удовлетворяющее неравенству (1.38). Следовательно, если нормы $\left\|u_{0}\right\|,\left\|v_{0}\right\|_{1}$ достаточно малы и таковы, что $\left\|v_{0}\right\|_{1}^{2}+C_{5}\left(\left\|u_{0}\right\|^{2}+\left\|v_{0}\right\|^{2}\right) \leqslant N$, то для найденного слабого решения выполнено неравенство (2.3), т.е. оно принадлежит $\mathscr{K}_{+}(N)$.

Рассмотрим трансляционную полугруппу $\{T(\tau)\}:=\{T(\tau), \tau \geqslant 0\}$, действующую в пространстве $\mathscr{F}_{+}^{\text {loc }}$ по формуле

$$
T(\tau)(y(t), z(t))=(y(t+\tau), z(t+\tau)), \quad t \geqslant 0 .
$$

Отметим, что полугруппа $\{T(\tau)\}$ переводит пространство траекторий $\mathscr{K}_{+}(N)$ в себя, т.е.

$$
T(\tau): \mathscr{K}_{+}(N) \rightarrow \mathscr{K}_{+}(N), \quad \tau \geqslant 0 .
$$

В самом деле, если $(u, v) \in \mathscr{K}_{+}(N)$, то функция

$$
T(\tau)(u(t), v(t))=(u(t+\tau), v(t+\tau))
$$

также является слабым решением автономной системы (1.1)-(1.2). Кроме того, поскольку $v(t)$ удовлетворяет (2.3), то

$$
\|v(t+\tau)\|_{1}^{2} \leqslant N \mathrm{e}^{-\sigma(t+\tau)}+R^{2} \leqslant N \mathrm{e}^{-\sigma t}+R^{2} .
$$


Поэтому $T(\tau) v(t)=v(t+\tau)$ также удовлетворяет (2.3), т.е. $T(\tau)(u, v) \in \mathscr{K}_{+}(N)$, $\tau \geqslant 0$.

УтВеРЖДЕНИЕ 2.1. Пространство траекторий $\mathscr{K}_{+}(N)$ принадлежит $\mathscr{F}_{+}^{\mathrm{b}}$, причем справедливо неравенство

$$
\|T(\tau)(u, v)\|_{\mathscr{F}_{+}^{\mathrm{b}}} \leqslant C_{6}\left(\|u(0)\|^{2}+N\right) \mathrm{e}^{-\rho \tau}+R_{3}^{2}, \quad \tau \geqslant 0
$$

где константы $C_{6} \geqslant 0$ и $\rho>0$ не зависят от $g_{1}, g_{2} u \delta$, а величина $R_{3}=$ $R_{3}\left(\left\|g_{1}\right\|,\left\|g_{2}\right\|\right)$ не зависит от $\delta$.

ДокАзАтельство. Применяя неравенства (1.27), (1.28), (2.3), находим, что

$$
\begin{aligned}
& \|T(\tau) u\|_{L_{\infty}\left(\mathbb{R}_{+} ; H\right)}^{2}+\|T(\tau) u\|_{L_{2}^{\mathrm{b}}\left(\mathbb{R}_{+} ; V\right)}^{p_{1}}+\|T(\tau) u\|_{L_{p_{1}}^{\mathrm{b}}\left(\mathbb{R}_{+} ; L_{p_{1}}\right)}^{2}+\|\|_{L_{\infty}\left(\mathbb{R}_{+} ; V\right)}+\|T(\tau) v\|_{L_{p_{2}}^{\mathrm{b}}\left(\mathbb{R}_{+} ; L_{p_{2}}\right)}^{p_{2}} \leqslant C_{7}\left(\|u(0)\|^{2}+N\right) \mathrm{e}^{-\rho_{1} \tau}+R_{4}^{2} \\
& \quad+\| T(\tau)
\end{aligned}
$$

для соответствующих $\rho_{1}$ и $R_{4}$. Осталось проверить аналогичные оценки для норм

$$
\left\|T(\tau) \partial_{t} u\right\|_{L_{q_{1}}^{\mathrm{b}}\left(\mathbb{R}_{+} ; H^{-r_{1}}\right)}, \quad\left\|T(\tau) \partial_{t} v\right\|_{L_{q_{2}}^{\mathrm{b}}\left(\mathbb{R}_{+} ; H^{-r_{2}}\right)} .
$$

Для этого воспользуемся уравнениями (1.1)-(1.2) и получим следующие оценки, например, для $\partial_{t} u$ :

$$
\begin{aligned}
& {\left[\int_{t}^{t+1}\left\|\partial_{t} u(s)\right\|_{H^{-r_{1}}}^{q_{1}} d s\right]^{1 / q_{1}}} \\
& \quad \leqslant\left[\int_{t}^{t+1}\|\Delta u(s)\|_{H^{-r_{1}}}^{q_{1}} d s\right]^{1 / q_{1}}+\left[\int_{t}^{t+1}\|f(u(s), v(s))\|_{H^{-r_{1}}}^{q_{1}} d s\right]^{1 / q_{1}}+\left\|g_{1}\right\|_{H^{-r_{1}}} \\
& \quad \leqslant C_{8}\left[\int_{t}^{t+1}\|u(s)\|_{1}^{2} d s\right]^{1 / 2}+C_{9}\left[\int_{t}^{t+1}\left(\|u(s)\|_{L_{p_{1}}}^{p_{1}}+\|v(s)\|_{L_{p_{2}}}^{p_{2}}\right) d s+1\right]^{1 / q_{1}}+\left\|g_{1}\right\| \\
& \quad \leqslant C_{10}\left(\|u(0)\|^{2}+N\right) \mathrm{e}^{-\rho_{2} \tau}+R_{5}^{2}, \quad t \geqslant \tau .
\end{aligned}
$$

Здесь мы снова воспользовались оценкой (1.28), выбором числа $r_{1}$, а также неравенством $q_{1} \leqslant 2$. Следовательно,

$$
\left\|T(\tau) \partial_{t} u\right\|_{L_{q_{1}}^{\mathrm{b}}\left(\mathbb{R}_{+} ; H^{-r_{1}}\right)} \leqslant C_{11}\left(\|u(0)\|^{2}+N\right) \mathrm{e}^{-\rho_{3} \tau}+R_{6}^{2}
$$

Аналогично, используя уравнение (1.2), можно доказать неравенство

$$
\left\|T(\tau) \partial_{t} v\right\|_{L_{q_{2}}^{\mathrm{b}}\left(\mathbb{R}_{+} ; H^{-r_{2}}\right)} \leqslant C_{12}\left(\|u(0)\|^{2}+N\right) \mathrm{e}^{-\rho_{4} \tau}+R_{7}^{2}
$$

Объединяя неравенства (2.6)-(2.8), получим оценку (2.5), из которой, в частности, следует, что $\mathscr{K}_{+}(N) \subset \mathscr{F}_{+}^{\mathrm{b}}$.

УтвеРЖДЕНИЕ 2.2. Пространство $\mathscr{K}_{+}(N)$ замкнуто в $\Theta_{+}^{\text {loc }}$ при любом $N \geqslant 0$.

ДокАЗАТЕЛЬство. Рассмотрим произвольную последовательность $w_{m}=$ $\left(u_{m}(t), v_{m}(t)\right)=:\left(u_{m}, v_{m}\right) \in \mathscr{K}_{+}(N), m=1,2, \ldots$, сходящуюся при $m \rightarrow \infty$ 
в топологии $\Theta_{+}^{\text {loc }}$ к функции $w=(u(t), v(t))=:(u, v) \in \mathscr{F}_{+}^{\text {loc }}$. Покажем, что $w \in \mathscr{K}_{+}(N)$. Указанная выше сходимость означает, что при $m \rightarrow \infty$ на любом интервале $[0, M](M>0)$ имеют место следующие сходимости:

$$
\left.\begin{array}{c}
u_{m}(\cdot) \rightarrow u(\cdot) \quad \text { *-слабо в } L_{\infty}(0, M ; H) \\
u_{m}(\cdot) \rightarrow u(\cdot) \\
v_{m}(\cdot) \rightarrow v(\cdot) \quad \text { слабо в } L_{2}(0, M ; V) \\
u_{m}(\cdot) \rightarrow u(\cdot) \quad \text { слабабо в } L_{\infty}(0, M ; V)
\end{array}\right\},
$$

Тогда, очевидно, последовательность функций $\left\{u_{m}\right\}$ ограничена в пространствах $L_{\infty}(0, M ; H), L_{2}(0, M ; V)$ и $L_{p_{1}}\left(0, M ; L_{p_{1}}(\Omega)\right)$, последовательность функций $\left\{v_{m}\right\}$ ограничена в $L_{\infty}(0, M ; V)$ и $L_{p_{2}}\left(0, M ; L_{p_{2}}(\Omega)\right)$, а последовательности $\left\{\partial_{t} u_{m}\right\}$ и $\left\{\partial_{t} v_{m}\right\}$ ограничены в $L_{q_{1}}\left(0, M ; H^{-r_{1}}(\Omega)\right)$ и $L_{q_{2}}\left(0, M ; H^{-r_{2}}(\Omega)\right)$ соответственно. Следовательно, в силу неравенства (1.13) последовательности $\left\{f\left(u_{m}, v_{m}\right)\right\}$ и $\left\{h\left(u_{m}, v_{m}\right)\right\}$ ограничены в пространствах $L_{q_{1}}\left(0, M ; L_{q_{1}}(\Omega)\right)$ и $L_{q_{2}}\left(0, M ; L_{q_{2}}(\Omega)\right)$ соответственно. Таким образом, переходя, если необходимо, к подпоследовательности $\left\{m^{\prime}\right\} \subset\{m\}$ и сохраняя для нее прежнее обозначение, можно считать, что при $m \rightarrow \infty$

$$
\left.\begin{array}{l}
f\left(u_{m}, v_{m}\right) \rightarrow \varphi(\cdot) \quad \text { слабо в } L_{q_{1}}\left(0, M ; L_{q_{1}}(\Omega)\right), \\
h\left(u_{m}, v_{m}\right) \rightarrow \chi(\cdot) \quad \text { слабо в } L_{q_{2}}\left(0, M ; L_{q_{2}}(\Omega)\right),
\end{array}\right\},
$$

где $\varphi=\varphi(x, t)$ и $\chi=\chi(x, t)$ - некоторые функции из пространств $L_{q_{1}}\left(0, M ; L_{q_{1}}(\Omega)\right)$ и $L_{q_{2}}\left(0, M ; L_{q_{2}}(\Omega)\right)$ соответственно.

Поскольку пара $\left(u_{m}(t), v_{m}(t)\right)$ является слабым решением, то эти функции удовлетворяют уравнениям (1.1)-(1.2) в смысле обобщенных функций, т.е.

$$
\begin{aligned}
& \partial_{t} u_{m}=\Delta u_{m}-f\left(u_{m}, v_{m}\right)+g_{1}(x), \\
& \partial_{t} v_{m}=\delta \Delta v_{m}-h\left(u_{m}, v_{m}\right)+g_{2}(x) .
\end{aligned}
$$

Тогда из свойств $(2.9),(2.11)$ и $(2.12)$ заключаем, что пара функций $(u(t), v(t))$ удовлетворяет в обобщенном смысле уравнениям

$$
\begin{aligned}
& \partial_{t} u=\Delta u-\varphi(x, t)+g_{1}(x), \\
& \partial_{t} v=\delta \Delta v-\chi(x, t)+g_{2}(x), \quad 0 \leqslant t \leqslant M .
\end{aligned}
$$

Напомним, что последовательность функций $\left\{u_{m}(t)\right\}$ ограничена в $L_{2}(0, M ; V)$, а последовательность производных $\left\{\partial_{t} u_{m}(t)\right\}$ ограничена в $L_{q_{1}}\left(0, M ; H^{-r_{1}}(\Omega)\right)$. Кроме того, вложение $V \Subset H \equiv L_{2}(\Omega)$ компактно. Поэтому согласно теореме Обена (см. [17], [18]) последовательность $\left\{u_{m}(t)\right\}$ образует предкомпактное множество в пространстве $L_{2}\left(0, M ; L_{2}(\Omega)\right)$. Следовательно, при $m \rightarrow \infty$

$$
u_{m}(\cdot) \rightarrow u(\cdot) \text { сильно в } L_{2}(\Omega \times] 0, M[) .
$$


Переходя опять к подпоследовательности и сохраняя прежнее обозначение, получаем, что при $m \rightarrow \infty$

$$
\left.u_{m}(x, t) \rightarrow u(x, t) \text { для почти всех }(x, t) \in \Omega \times\right] 0, M[.
$$

Аналогично убеждаемся в том, что при $m \rightarrow \infty$

$$
\left.v_{m}(x, t) \rightarrow v(x, t) \text { для почти всех }(x, t) \in \Omega \times\right] 0, M[.
$$

Тогда в силу непрерывности функций $f$ и $h$ получаем, что при $m \rightarrow \infty$

$$
\begin{aligned}
& f\left(u_{m}(x, t), v_{m}(x, t)\right) \rightarrow f(u(x, t), v(x, t)), \\
& h\left(u_{m}(x, t), v_{m}(x, t)\right) \rightarrow h(u(x, t), v(x, t))
\end{aligned}
$$

для почти всех $(x, t) \in \Omega \times] 0, M\left[\right.$. Последовательности $\left\{f\left(u_{m}, v_{m}\right)\right\},\left\{h\left(u_{m}, v_{m}\right)\right\}$ ограничены в $L_{q_{1}}\left(0, M ; L_{q_{1}}(\Omega)\right)$ и $L_{q_{2}}\left(0, M ; L_{q_{2}}(\Omega)\right)$ соответственно. Теперь можно применить известную лемму Лионса о слабой сходимости (см. [14; гл. 1 , лемма 1.3]), из которой получаются следующие сходимости при $m \rightarrow \infty$ :

$$
\begin{aligned}
& f\left(u_{m}, v_{m}\right) \rightarrow f(u, v) \text { слабо в } L_{q_{1}}\left(0, M ; L_{q_{1}}(\Omega)\right), \\
& h\left(u_{m}, v_{m}\right) \rightarrow h(u, v) \text { слабо в } L_{q_{2}}\left(0, M ; L_{q_{2}}(\Omega)\right) .
\end{aligned}
$$

Но тогда из (2.12) заключаем, что

$$
\varphi(x, t) \equiv f(u(x, t), v(x, t)), \quad \chi(x, t) \equiv h(u(x, t), v(x, t))
$$

почти всюду в $\Omega \times] 0, M[$. Таким образом, пара $(u(x, t), v(x, t))$ является слабым решением системы (1.1)-(1.2). Осталось проверить, что для функции $v(x, t)$ выполнено неравенство (2.3). Действительно, функции $v_{m}(x, t)$ удовлетворяют (2.3). Тогда для любого $\theta \geqslant 0$

$$
\operatorname{ess} \sup \left\{\left\|v_{m}(\theta)\right\|_{1}^{2} \mid t \leqslant \theta \leqslant t+1\right\} \leqslant N \mathrm{e}^{-\sigma t}+R^{2}, \quad m \in \mathbb{N} .
$$

Напомним, что

$$
v_{m}(\cdot) \neg v(\cdot) * \text {-слабо в } L_{\infty}(0, M ; V), \quad M>0 .
$$

Таким образом, при каждом $t \geqslant 0$

$$
\begin{aligned}
& \operatorname{ess} \sup \left\{\|v(\theta)\|_{1}^{2} \mid t \leqslant \theta \leqslant t+1\right\} \\
& \quad \leqslant \underline{\varliminf_{m \rightarrow \infty}}\left\{\left\|v_{m}(\theta)\right\|_{1}^{2} \mid t \leqslant \theta \leqslant t+1\right\} \leqslant N \mathrm{e}^{-\sigma t}+R^{2} .
\end{aligned}
$$

Из леммы Лионса-Мадженеса получаем, что $v(\cdot) \in C_{w}\left(\mathbb{R}_{+} ; V\right)$. Следовательно, функция $\|v(t)\|_{1}, t \geqslant 0$, является полунепрерывной снизу, т.е., в частности,

$$
\|v(t)\|_{1} \leqslant \underline{\lim _{\theta \rightarrow t^{+}}}\|v(\theta)\|_{1}
$$

(см., например, [3]). Используя это свойство вместе с (2.19), находим, что

$$
\|v(t)\|_{1} \leqslant N \mathrm{e}^{-\sigma t}+R^{2}, \quad t \geqslant 0 .
$$

Неравенство (2.3) установлено для функции $v(x, t)$ и, следовательно, $w=(u, v) \in$

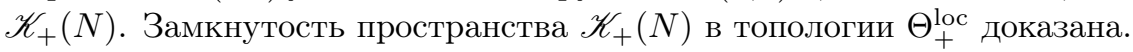


Вернемся к исследованию трансляционной полугруппы $\{T(\tau)\}$, действующей на пространстве траекторий $\mathscr{K}_{+}(N)$.

ОПРЕДЕЛЕНИЕ 2.2. Множество $\mathscr{P} \subseteq \mathscr{K}_{+}(N)$ называется поглощающим для полугруппы $\{T(\tau)\}$, если для любого множества $B \subset \mathscr{K}_{+}(N)$, ограниченного в $\mathscr{F}_{+}^{\mathrm{b}}$, найдется число $\tau_{1}=\tau_{1}(B) \geqslant 0$ такое, что $T(\tau) B \subseteq \mathscr{P}, \tau \geqslant \tau_{1}$.

ОПРЕДЕЛЕНИЕ 2.3. Множество $\mathscr{P} \subseteq \mathscr{K}_{+}(N)$ называется притягивающим для полугруппы $\{T(\tau)\}$, если любая окрестность $\mathscr{O}(\mathscr{P})$ множества $\mathscr{P}$ в топологии $\Theta_{+}^{\text {loc }}$ является поглощающим множеством, т.е. для любого множества $B \subset \mathscr{K}_{+}(N)$, ограниченного в $\mathscr{F}_{+}^{\mathrm{b}}$, найдется число $\tau_{1}=\tau_{1}(B, \mathscr{O}) \geqslant 0$ такое, что $T(\tau) B \subseteq \mathscr{O}(\mathscr{P}), \tau \geqslant \tau_{1}$.

Ясно, что любое поглощающее множество является притягивающим. Дадим основное определение траекторного аттрактора полугруппы $\{T(\tau)\}$.

ОПРедЕЛЕНИЕ 2.4. Множество $\mathfrak{A} \subset \mathscr{K}_{+}(N)$ называется траекторным аттрактором полугруппы $\{T(\tau)\}$ на $\mathscr{K}_{+}(N)$, если оно ограничено в $\mathscr{F}_{+}^{\mathrm{b}}$, компактно в топологии $\Theta_{+}^{\text {loc }}$, строго инвариантно относительно $\{T(\tau)\}$, т.е.

$$
T(\tau) \mathfrak{A}=\mathfrak{A}, \quad \tau \geqslant 0,
$$

и является притягивающим множеством $\{T(\tau)\}$ на $\mathscr{K}_{+}(N)$ в топологии $\Theta_{+}^{\text {loc }}$.

Легко видеть, что траекторный аттрактор трансляционной полугруппы определен однозначно. Наша цель - построить траекторный аттрактор $\mathfrak{A}$ полугруппы $\{T(\tau)\}$ на $\mathscr{K}_{+}(N)$ и исследовать его свойства. Множество $\mathfrak{A}$ называется также траекторным аттрактором системы (1.1)-(1.2).

Из неравенства (2.5) получаем, что множество

$$
P=\left\{(u, v) \in \mathscr{K}_{+}(N) \mid\|(u, v)\|_{\mathscr{F}_{+}^{\mathrm{b}}} \leqslant 2 R_{3}^{2}\right\}
$$

является поглощающим для полугруппы $\{T(\tau)\}$ в пространстве $\mathscr{K}_{+}(N)$. Множество $P$ является ограниченным в $\mathscr{F}_{+}^{\mathrm{b}}$. Рассмотрим на $P$ топологию, индуцированную из $\Theta_{+}^{\mathrm{loc}}$. Это топологическое пространство является компактным и метризуемым (см. замечание 2.1). Из (2.4), и из очевидного неравенства

$$
\|T(\tau)(u, v)\|_{\mathscr{F}_{+}^{\mathrm{b}}} \leqslant\|(u, v)\|_{\mathscr{F}_{+}^{\mathrm{b}}}, \quad \tau \geqslant 0,
$$

следует, что полугруппа $\{T(\tau)\}$ отображает $P$ в себя, т.е.

$$
T(\tau) P \subseteq P, \quad \tau \geqslant 0 .
$$

Легко доказать, что полугруппа $\{T(\tau)\}$ непрерывна на $\mathscr{K}_{+}(N)$ в топологии $\Theta_{+}^{\text {loc }}$. Таким образом, имеется непрерывная полугруппа $\{T(\tau)\}$ на компактном метрическом пространстве $P$. Тогда применима общая теорема о существовании у полугруппы $\{T(\tau)\}$ глобального аттрактора $\mathfrak{A}(N) \subseteq P$ (см., например, [1], [2], [4]), который задается формулой

$$
\mathfrak{A}(N)=\bigcap_{\tau \geqslant 0}\left[\bigcup_{\theta \geqslant \tau} T(\theta) P\right]_{\Theta_{+}^{\text {loc }}} .
$$


Множество $\mathfrak{A}(N)$ обладает следующими свойствами: оно ограничено в $\mathscr{F}_{+}^{\mathrm{b}}$, компактно в $\Theta_{+}^{\text {loc }}$, строго инвариантно, т.е.

$$
T(\tau) \mathfrak{A}(N)=\mathfrak{A}(N), \quad \tau \geqslant 0,
$$

и, будучи глобальным аттрактором, является притягивающим для любого множества $B \subseteq P$. Однако $P$ является поглощающим множеством полугруппы в $\{T(\tau)\}$, поэтому $\mathfrak{A}(N)$ притягивает любое ограниченное множество $B \subset$ $\mathscr{K}_{+}(N)$. Следовательно, $\mathfrak{A}(N)$ - траекторный аттрактор $\{T(\tau)\}$ в $\mathscr{K}_{+}(N)$.

УтВЕРЖДЕНИЕ 2.3. Построенный траекторный аттрактор не зависит от $N$, т.е. $\mathfrak{A}(N)=\mathfrak{A}$. В частности, $\mathfrak{A}=\mathfrak{A}(0)$, а именно,

$$
\sup \left\{\|v(t)\|_{1}^{2} \mid t \geqslant 0\right\} \leqslant R^{2}, \quad(u, v) \in \mathfrak{A}
$$

ДоказАтельство. Пусть $N>0$. Из определения пространства $\mathscr{K}_{+}(N)$ следует, что $\mathscr{K}_{+}(N) \subseteq \mathscr{K}_{+}\left(N_{1}\right)$ при $N_{1} \geqslant N$. Поэтому $\mathfrak{A}(N) \subseteq \mathfrak{A}\left(N_{1}\right)$ при $N_{1} \geqslant N$. Из (2.3) получаем, что $T(\tau) \mathscr{K}_{+}\left(N_{1}\right) \subseteq \mathscr{K}_{+}(N)$ при $\tau \geqslant \sigma^{-1} \ln \left(N_{1} / N\right)$ и, в частности, $T(\tau) \mathfrak{A}\left(N_{1}\right) \subseteq \mathscr{K}_{+}(N)$. В силу строгой инвариантности аттрактора имеем $\mathfrak{A}\left(N_{1}\right)=T(\tau) \mathfrak{A}\left(N_{1}\right)$, т.е. $\mathfrak{A}\left(N_{1}\right) \subseteq \mathscr{K}_{+}(N)$. Но тогда опять из строгой инвариантности аттракторов следует, что $\mathfrak{A}\left(N_{1}\right) \subseteq \mathfrak{A}(N)$ при всех $N_{1} \geqslant N$. Итак, $\mathfrak{A}\left(N_{1}\right)=\mathfrak{A}(N)$ при $N_{1} \geqslant N$. Мы установили, что $\mathfrak{A}=\mathfrak{A}(N)$ не зависит от $N$ при $N>0$, т.е. для любого $(u, v) \in \mathfrak{A}$ справедливо неравенство

$$
\sup \left\{\|v(t)\|_{1}^{2} \mid t \geqslant 0\right\} \leqslant N+R^{2}, \quad N>0 .
$$

Следовательно, имеет место (2.22).

В заключение опишем структуру траекторного аттрактора $\mathfrak{A}$ с помощью полных траекторий системы (1.1)-(1.2), т.е. слабых решений этой системы, заданных на всей оси времени.

Пространства $\mathscr{F}^{\mathrm{loc}}, \mathscr{F}^{\mathrm{b}}$ и топология $\Theta^{\mathrm{loc}}$ вводятся аналогично пространствам $\mathscr{F}_{+}^{\text {loc }}, \mathscr{F}_{+}^{\mathrm{b}}$ и топологии $\Theta_{+}^{\text {loc }}$ с заменой в их определениях полупрямой $\mathbb{R}_{+}$ $(t \geqslant 0)$ на всю числовою прямую $\mathbb{R}(-\infty<t<\infty)$. В частности,

$$
\mathscr{F}^{\mathrm{loc}}=\left\{\begin{array}{c}
(y(x, t), z(x, t)), x \in \Omega, t \in \mathbb{R} \mid \\
y \in L_{\infty}^{\mathrm{loc}}(\mathbb{R} ; H) \cap L_{2}^{\mathrm{loc}}(\mathbb{R} ; V) \cap L_{p_{1}}^{\mathrm{loc}}\left(\mathbb{R} ; L_{p_{1}}(\Omega)\right), \\
z \in L_{\infty}^{\mathrm{loc}}(\mathbb{R} ; V) \cap L_{p_{2}}^{\mathrm{loc}}\left(\mathbb{R} ; L_{p_{2}}(\Omega)\right), \\
\partial_{t} y \in L_{q_{1}}^{\mathrm{loc}}\left(\mathbb{R} ; H^{-r_{1}}(\Omega)\right), \partial_{t} z \in L_{q_{2}}^{\text {loc }}\left(\mathbb{R} ; H^{-r_{2}}(\Omega)\right)
\end{array}\right\}
$$

(см. (2.1)), а норма в пространстве $\mathscr{F}$ b задается по формуле (ср. с (2.2))

$$
\begin{gathered}
\|(y, z)\|_{\mathscr{F} \mathrm{b}}:=\|y\|_{L_{\infty}(\mathbb{R} ; H)}+\|y\|_{L_{2}^{\mathrm{b}}(\mathbb{R} ; V)}+\|y\|_{L_{p_{1}}^{\mathrm{b}}\left(\mathbb{R} ; L_{p_{1}}\right)}+\left\|\partial_{t} y\right\|_{L_{q_{1}}^{\mathrm{b}}\left(\mathbb{R} ; H^{-r_{1}}\right)} \\
+\|z\|_{L_{\infty}(\mathbb{R} ; V)}+\|z\|_{L_{p_{2}}^{\mathrm{b}}\left(\mathbb{R} ; L_{p_{2}}\right)}+\left\|\partial_{t} z\right\|_{L_{q_{2}}^{\mathrm{b}}\left(\mathbb{R} ; H^{-r_{2}}\right)} .
\end{gathered}
$$

Рассматриваются слабые решения $\{u(t), v(t)\}, t \in \mathbb{R}$, системы (1.1)-(1.2), которые принадлежат пространству $\mathscr{F}^{\text {loc }}$. 
ОПРЕДЕЛЕНИЕ 2.5. Ядром $\mathscr{K}$ системы уравнений (1.1)-(1.2) в пространстве $\mathscr{F}^{\mathrm{b}}$ называется множество слабых решений $\{u(t), v(t)\}, t \in \mathbb{R}$, этой системы из $\mathscr{F}^{\mathrm{loc}}$, принадлежащих $\mathscr{F}^{\mathrm{b}}$ (т.е. имеющих конечную норму $\|(u, v)\|_{\mathscr{F}}$, определенную в (2.24)), и для которых выполнено неравенство

$$
\sup \left\{\|v(t)\|_{1} \mid t \in \mathbb{R}\right\} \leqslant R,
$$

где величина $R$ взята из (2.3).

Обозначим через $\Pi_{+}$оператор ограничения на полуось $\mathbb{R}_{+}$функций, заданных на всей оси $\mathbb{R}$, который функции $f(t), t \in \mathbb{R}$, ставит в соответствие функцию $\Pi_{+} f(t), t \geqslant 0$, где $\Pi_{+} f(t) \equiv f(t)$ при $t \geqslant 0$.

Справедлива следующая основная теорема.

ТеОрема 2.1. Ядро $\mathscr{K}$ системы (1.1)-(1.2) ограничено в пространстве $\mathscr{F} \mathrm{b}$ и компактно в топологии $\Theta^{\mathrm{loc}}$. Траекторный аттрактор $\mathfrak{A}$ этой системы (1.1)-(1.2) совпадает с ограничением на $\mathbb{R}_{+}$ядра $\mathscr{K}$, m.е.

$$
\mathfrak{A}=\Pi_{+} \mathscr{K} .
$$

ДокАЗАтельство. Действительно, пусть $\zeta=(y, z) \in \mathscr{K}$. Покажем, что $\Pi_{+} \zeta \in \mathfrak{A}$. Рассмотрим множество $B_{\zeta}=\left\{\Pi_{+} \zeta(\eta+t) \mid \eta \in \mathbb{R}\right\}$, которому принадлежит $\zeta$. Каждая пара функций $\Pi_{+} \zeta(\eta+t)=(y(\eta+t), z(\eta+t)), t \geqslant 0$, является слабым решением системы (1.1)-(1.2). Кроме того, в силу (2.25) имеем $B_{\zeta} \subseteq \mathscr{K}_{+}(0)$, причем множество $B_{\zeta}$ ограничено в $\mathscr{F}_{+}^{\mathrm{b}}$ и строго инвариантно относительно полугруппы $\{S(\tau)\}$, так как, очевидно $S(\tau) B_{\zeta}=B_{\zeta}, \tau \geqslant 0$. Следовательно,

$$
B_{\zeta} \subseteq \mathfrak{A}(0)
$$

и, в частности, при всех $\zeta \in \mathscr{K}$ функция $\Pi_{+} \zeta \in \mathfrak{A}(0) \equiv \mathfrak{A}$ (см. утверждение 2.3). Итак, мы показали, что

$$
\Pi_{+} \mathscr{K} \subseteq \mathfrak{A} .
$$

Установим теперь обратное включение. Для этого необходимо доказать, что любое слабое решение $(u(t), v(t)), t \geqslant 0$, системы $(1.1)-(1.2)$, принадлежащее $\mathfrak{A}=\mathfrak{A}(0)$, можно продолжить как решение на отрицательную полупрямую с сохранением неравенства (2.25) при всех $t \in \mathbb{R}$.

В самом деле, из свойства строгой инвариантности множества $\mathfrak{A}$ следует, что для любого $(u, v) \in \mathfrak{A}$ найдется слабое решение $\left(u_{1}(t), v_{1}(t)\right), t \geqslant 0$, такое, что $\left(u_{1}, v_{1}\right) \in \mathfrak{A} \subseteq \mathscr{K}_{+}(0)$ и $\left(u_{1}(t+1), v_{1}(t+1)\right)=(u(t), v(t)), t \geqslant 0$. Обозначим $(\widetilde{u}(t), \widetilde{v}(t))=\left(u_{1}(t+1), v_{1}(t+1)\right)$ при $t \geqslant-1$. Ясно, что $(\widetilde{u}(t), \widetilde{v}(t))$ является слабым решением при $t \geqslant-1$. Эта пара совпадает с $(u(t), v(t))$ при $t \geqslant 0$ и

$$
\sup \left\{\|v(t)\|_{1} \mid t \geqslant-1\right\} \leqslant R
$$

Повторяя этот процесс для $\left(u_{1}, v_{1}\right)$ вместо $(u, v)$, продолжаем выбранное решение на полупрямую $\{t \geqslant-2\}$ с сохранением нужных свойств, потом на $\{t \geqslant-3\}$ и т.д. В результате будет построено полное слабое решение $(\widetilde{u}(t), \widetilde{v}(t)), t \in \mathbb{R}$, системы (1.1)-(1.2), удовлетворяющее неравенству

$$
\sup \left\{\|v(t)\|_{1} \mid t \in \mathbb{R}\right\} \leqslant R,
$$


которое принадлежит $\mathscr{F}^{\mathrm{b}}$, так как $\mathfrak{A}$ было ограничено в $\mathscr{F}_{+}^{\mathrm{b}}$. Тогда $(\widetilde{u}, \widetilde{v}) \in \mathscr{K}$, причем $\Pi_{+}(\widetilde{u}, \widetilde{v})=(u, v)$. Следовательно, $(u, v) \in \Pi_{+} \mathscr{K}$ для всех $(u, v) \in \mathfrak{A}$, т.е.

$$
\mathfrak{A} \subseteq \Pi_{+} \mathscr{K},
$$

и тождество (2.26) установлено.

В дальнейшем будем использовать следующие обозначения:

$$
\mathfrak{A}^{\delta}:=\mathfrak{A}, \quad \mathscr{K}^{\delta}:=\mathscr{K},
$$

где $\delta$ - это коэффициент диффузии в уравнении (1.2). В следующих параграфах исследуется поведение множеств $\mathfrak{A}^{\delta}$ и $\mathscr{K}^{\delta}$ при $\delta \rightarrow 0^{+}$.

СледСтвиЕ 2.1. Семейство траекторных аттракторов $\left\{\mathfrak{A}^{\delta}, 0<\delta \leqslant 1\right\}$ равномерно ограничено по норме пространства $\mathscr{F}_{+}^{\mathrm{b}}$, a семейство ядер $\left\{\mathscr{K}^{\delta}, 0<\right.$ $\delta \leqslant 1\}$ равномерно ограничено в $\mathscr{F}^{\mathrm{b}}$.

Чтобы убедиться в этом, достаточно заметить, что в неравенстве $(2.5)$ величины $C_{6}, R_{3}$ и $\rho$ не зависят от $\delta$. Поэтому поглощающие множества $P^{\delta}$ для пространств $\mathscr{K}_{+}^{\delta}(N)$ равномерно ограничены в $\mathscr{F}_{+}^{\mathrm{b}}$ при $0<\delta \leqslant 1$. Отсюда в силу очевидных включений $\mathfrak{A}^{\delta} \subset P^{\delta}$ следует равномерная ограниченность аттракторов $\mathfrak{A}^{\delta}$ в $\mathscr{F}_{+}^{\mathrm{b}}$ при $0<\delta \leqslant 1$. Тогда равномерная ограниченность семейства ядер $\left\{\mathscr{K}^{\delta}, 0<\delta \leqslant 1\right\}$ в пространстве $\mathscr{F}^{\mathrm{b}}$ следует из представления $(2.26)$.

\section{§ 3. Предельная система уравнений, у которой один коэффициент диффузии равен нулю, и ее траекторный аттрактор}

Рассматривается предельная система уравнений системы (1.1)-(1.2), в которой коэффициент диффузии $\delta=0$ :

$$
\begin{aligned}
& \partial_{t} u=\Delta u-f(u, v)+g_{1}(x), \\
& \partial_{t} v=-h(u, v)+g_{2}(x) .
\end{aligned}
$$

Как и прежде, на границе области $\partial \Omega$ задаются условия Дирихле

$$
\left.u\right|_{\partial \Omega}=0,\left.\quad v\right|_{\partial \Omega}=0 .
$$

Все обозначения имеют тот же смысл, что и в $\S 1$. В частности, функции $f$ и $h$ удовлетворяют условиям (1.4)-(1.8), а функции $g_{1}$ и $g_{2}$ удовлетворяют условиям (1.9).

Слабым решением системы (3.1)-(3.2) в области $\Omega \times \mathbb{R}_{+}$называется пара функций $(u(x, t), v(x, t))$ таких, что

$$
\begin{aligned}
& u \in L_{p_{1}}^{\mathrm{loc}}\left(\mathbb{R}_{+} ; L_{p_{1}}(\Omega)\right) \cap L_{2}^{\mathrm{loc}}\left(\mathbb{R}_{+} ; V\right), \\
& v \in L_{p_{2}}^{\mathrm{loc}}\left(\mathbb{R}_{+} ; L_{p_{2}}(\Omega)\right) \cap L_{\infty}^{\mathrm{loc}}\left(\mathbb{R}_{+} ; V\right)
\end{aligned}
$$

и удовлетворяющих уравнениям (3.1)-(3.2) в пространстве обобщенных функций $\mathscr{D}^{\prime}\left(\mathbb{R}_{+} ; H^{-r_{1}}(\Omega) \times H^{-r_{2}}(\Omega)\right)$ (показатели $r_{1}$ и $r_{2}$ определены в $\S 1$ ). 
ЗАмЕчАниЕ 3.1. В отличие от случая $\delta>0$, рассмотренного $\S 1$, для компоненты $v(x, t)$ слабого решения при $\delta=0$ выбрано пространство $L_{\infty}^{\text {loc }}\left(\mathbb{R}_{+} ; V\right)$, а не $L_{2}^{\text {loc }}\left(\mathbb{R}_{+} ; V\right)$. Это связано с тем, что слабое решение для случая $\delta=0$ удается построить лишь при условии $v_{0} \in V$, применяя метод Галёркина и специальную априорную оценку.

Как показано в 1 , при указанном выше выборе пространств для слабых решений обобщенные производные $\partial_{t} u(x, t)$ и $\partial_{t} v(x, t)$ принадлежат пространствам

$$
\partial_{t} u \in L_{q_{1}}^{\text {loc }}\left(\mathbb{R}_{+} ; H^{-r_{1}}(\Omega)\right), \quad \partial_{t} v \in L_{q_{2}}^{\text {loc }}\left(\mathbb{R}_{+} ; H^{-r_{2}}(\Omega)\right) .
$$

Из леммы Лионса-Мадженеса следует, что

$$
u(\cdot) \in C_{w}\left(\mathbb{R}_{+} ; H\right), \quad v(\cdot) \in C_{w}\left(\mathbb{R}_{+} ; V\right) .
$$

Поэтому значения $u(t)$ и $v(t)$ определены при всех $t \geqslant 0$ и для уравнений (3.1)-(3.2) допустимы начальные данные вида

$$
\begin{aligned}
& \left.u\right|_{t=0}=u_{0} \in H, \\
& \left.v\right|_{t=0}=v_{0} \in V .
\end{aligned}
$$

Существование слабых решений задачи (3.1)-(3.3), (3.6), (3.7) доказывается по методу Галёркина. Стандартным образом устанавливается, что у последовательности галёркинских приближений $\left\{\left(u_{m}(x, t), v_{m}(x, t)\right)\right\}$ порядка $m=$ $1,2, \ldots$ имеется подпоследовательность, которая сходится в слабом смысле к некоторому слабому решению $(u(x, t), v(x, t))$, принадлежащему описанному выше функциональному классу. При этом используются априорные оценки, аналогичные оценкам, полученным в $\S 1$ для случая $\delta>0$.

Отметим, что любое слабое решение системы (3.1)-(3.2) удовлетворяет следующему энергетическому тождеству:

$$
\begin{gathered}
\frac{1}{2} \frac{d}{d t}\left\{\|u(t)\|^{2}+\|v(t)\|^{2}\right\}+\|\nabla u(t)\|^{2}+\int_{\Omega} f(u(x, t), v(x, t)) u(x, t) d x \\
+\int_{\Omega} h(u(x, t), v(x, t)) v(x, t) d x=\left\langle g_{1}, u(t)\right\rangle+\left\langle g_{2}, v(t)\right\rangle,
\end{gathered}
$$

которое устанавливается аналогично (1.18). Из этого равенства выводятся следующие оценки.

УТВеРЖДЕНИЕ 3.1. Для любого слабого решения $(u(t), v(t)), t \geqslant 0$, задачи (3.1)-(3.7) справедливы неравенства

$$
\begin{gathered}
\|u(t)\|^{2}+\|v(t)\|^{2}+2 \int_{0}^{t}\|\nabla u(s)\|^{2} \mathrm{e}^{-\sigma(t-s)} d s \leqslant\left(\left\|u_{0}\right\|^{2}+\left\|v_{0}\right\|^{2}\right) \mathrm{e}^{-\sigma t}+R_{1}^{2} \\
2 \int_{t}^{t+1}\|u(s)\|_{V}^{2} d s+\sigma \int_{t}^{t+1}\left\{\|u(s)\|_{L_{p_{1}}}^{p_{1}}+\|v(s)\|_{L_{p_{2}}}^{p_{2}}\right\} d s \\
\leqslant\left(\left\|u_{0}\right\|^{2}+\left\|v_{0}\right\|^{2}\right) \mathrm{e}^{-\sigma t}+R_{2}^{2}, \quad t \geqslant 0 .
\end{gathered}
$$

Величины $R_{1}$ и $R_{2}$ такие же, как и в утверждении 1.1 (см. (1.23) и (1.26)). 
Доказательство утверждения 3.1 аналогично доказательству приведенного выше утверждения 1.1 .

Отметим, что для построения слабых решений задачи (3.1)-(3.3), (3.6), (3.7) методом Галёркина априорных оценок вида (3.9) и (3.10) не хватает! В этом заключается существенное отличие предельной системы при $\delta=0$ от системы (1.1)-(1.2) при $\delta>0$, для которой априорных оценок (1.27) и (1.28) вполне достаточно для построения соответствующего слабого решения. Для случая $\delta=0$ такое построение возможно, если доказать специальную априорную оценку. Для этого надо учесть гладкость начального условия (3.7) и воспользоваться равенством

$$
\frac{1}{2} \frac{d}{d t}\left(\left\|v_{m}(t)\right\|_{1}^{2}\right)-\int_{\Omega} h\left(u_{m}(x, t), v_{m}(x, t)\right) \Delta v_{m}(x, t) d x=\left\langle\nabla g_{2}, \nabla v_{m}(t)\right\rangle,
$$

аналогичным (1.30), которое справедливо при $t \geqslant 0$ для любого галёркинского приближения $\left(u_{m}(x, t), v_{m}(x, t)\right)$ задачи (3.1)-(3.3), (3.6), (3.7). Используя тождество (3.11) и рассуждая так же, как и при доказательстве утверждения 1.2 , доказываем

УтвеРЖДЕНИЕ 3.2. Задача (3.1)-(3.3), (3.6), (3.7) имеет слабое решение $(u(t), v(t))$ в классах функций (3.4)-(3.5), удовлетворяющее неравенству

$$
\|v(t)\|_{1}^{2} \leqslant\left\|v_{0}\right\|_{1}^{2} \mathrm{e}^{-\sigma t}+C_{5}\left(\left\|u_{0}\right\|^{2}+\left\|v_{0}\right\|^{2}\right) \mathrm{e}^{-\sigma t}+R^{2}, \quad t \geqslant 0,
$$

где величина $R$ и константа $C_{5}$ такие же, как и в утверждении 1.2 .

Построим траекторный аттрактор предельной системы (3.1)-(3.2). Будем использовать пространства $\mathscr{F}_{+}^{\text {loc }}, \mathscr{F}_{+}^{\mathrm{b}}$ и топологию $\Theta_{+}^{\mathrm{loc}}$, которые введены в начале $\S 2$.

Траекторное пространство $\mathscr{K}_{+}^{0}(N)$ для системы (3.1)-(3.2) вводится аналогично пространствам $\mathscr{K}_{+}^{\delta}(N):=\mathscr{K}_{+}(N)$ системы (1.1)-(1.2) (см. определение 2.1).

ОПРЕДЕЛЕНИЕ 3.1. Пространство $\mathscr{K}_{+}^{0}(N)$ состоит из функций $(u(\cdot), v(\cdot)) \in$ $\mathscr{F}_{+}^{\text {loc }}$ таких, что

(i) $(u(t), v(t)), t \geqslant 0$, является слабым решением системы (3.1)-(3.2);

(ii) функция $v(t)$ удовлетворят неравенству

$$
\|v(t)\|_{1}^{2} \leqslant N \mathrm{e}^{-\sigma t}+R^{2}, \quad t \geqslant 0
$$

где величины $\sigma$ и $R$ взяты из неравенства (1.38).

С помощью утверждения 3.2 убеждаемся в том, что пространство $\mathscr{K}_{+}^{0}(N)$ не пусто и достаточно широко при любом $N>0$.

Для пространств $\mathscr{K}_{+}^{0}(N)$ справедливы утверждения 2.1 и 2.2 , поскольку при их доказательствах условие $\delta>0$ не использовалось, а все константы и величины, участвовавшие в оценках, не зависели от $\delta$. Значит, все рассуждения остаются справедливыми и при $\delta=0$, если принять во внимание утверждения 3.1 и 3.2 .

С помощью утверждения 2.1 устанавливается, что множество

$$
P^{0}=\left\{(u, v) \in \mathscr{K}_{+}^{0}(N) \mid\|(u, v)\|_{\mathscr{F}_{+}^{\mathrm{b}}} \leqslant 2 R_{3}^{2}\right\}
$$


является поглощающим для трансляционной полугруппы $\{T(\tau)\}$ в пространстве $\mathscr{K}_{+}^{0}(N)$. Множество $P^{0}$ является метрическим пространством в топологии, индуцированной топологией $\Theta_{+}^{\text {loc }}$, и, кроме того, $P^{0}$ ограничено по норме $\mathscr{F}_{+}^{\mathrm{b}}$.

Траекторный аттрактор $\mathfrak{A}^{0}$ системы (3.1)-(3.2) строится по формуле

$$
\mathfrak{A}^{0}=\bigcap_{\tau \geqslant 0}\left[\bigcup_{\theta \geqslant \tau} T(\theta) P^{0}\right]_{\Theta_{+}^{\text {loc }}} .
$$

Множество $\mathfrak{A}^{0}$ не зависит от $N$ и для него справедливо неравенство

$$
\sup \left\{\|v(t)\|_{1}^{2} \mid t \geqslant 0\right\} \leqslant R^{2}, \quad(u, v) \in \mathfrak{A}^{0} .
$$

Доказательство этой оценки аналогично доказательству утверждения 2.3.

Наконец, пусть $\mathscr{K}^{0}$ обозначает ядро системы (3.1)-(3.2), состоящее из всех ее слабых решений $(u(t), v(t))$, заданных при всех $t \in \mathbb{R}$, ограниченных по норме $\mathscr{F}^{\mathrm{b}}$, и для которых выполнено неравенство

$$
\sup \left\{\|v(t)\|_{1} \mid t \in \mathbb{R}\right\} \leqslant R,
$$

где величина $R$ взята из (2.3). При помощи рассуждений, аналогичных тем, что приведены в доказательстве теоремы 2.1, устанавливается равенство

$$
\mathfrak{A}^{0}=\Pi_{+} \mathscr{K}^{0},
$$

которое будет использоваться в следующем параграфе.

\section{§4. Предел решений и траекторных аттракторов $\mathfrak{A}^{\delta}$ системы реакции-диффузии при $\delta \rightarrow 0^{+}$}

Рассмотрим последовательность слабых решений $\left\{\left(y_{m}(t), z_{m}(t)\right)\right\}, t \geqslant 0$, систем (1.1)-(1.2) с коэффициентами диффузии $\delta=\delta_{m}>0$, принадлежащими пространствам траекторий $\mathscr{K}_{+}^{\delta_{m}}(0)$.

УтВЕРЖДЕНИЕ 4.1. Если последовательность $\left\{\left(y_{m}(t), z_{m}(t)\right), t \geqslant 0\right\}$ ограничена в пространстве $\mathscr{F}_{+}^{\mathrm{b}}$,

$$
\begin{gathered}
\left(y_{m}, z_{m}\right) \in \mathscr{K}_{+}^{\delta_{m}}(0), \\
\delta_{m} \rightarrow 0^{+}, \quad m \rightarrow \infty,
\end{gathered}
$$

то найдутся подпоследовательность индексов $\left\{m^{\prime}\right\} \subset\{m\}$ и пара функиий

$$
(y, z) \in \mathscr{F}_{+}^{\mathrm{b}}
$$

такие, что при $\mathrm{m}^{\prime} \rightarrow \infty$

$$
\left(y^{m^{\prime}}, z^{m^{\prime}}\right) \rightarrow(y, z) \text { в } \Theta_{+}^{\text {loc }},
$$

причем $(y(t), z(t)), t \geqslant 0$, является слабым решением системы (3.1)-(3.2) и

$$
(y, z) \in \mathscr{K}_{+}^{0}(0) .
$$


ДокАзАтЕЛЬСтво. Функции $\left(y_{m}(t), z_{m}(t)\right)$ удовлетворяют системе уравнений

$$
\begin{aligned}
& \partial_{t} y_{m}=\Delta y_{m}-f\left(y_{m}, z_{m}\right)+g_{1}(x), \\
& \partial_{t} z_{m}=\delta_{m} \Delta z_{m}-h\left(y_{m}, z_{m}\right)+g_{2}(x) .
\end{aligned}
$$

Ограниченная в $\mathscr{F}_{+}^{\mathrm{b}}$ последовательность $\left\{\left(y_{m}(\cdot), z_{m}(\cdot)\right)\right\}$ является компактной в топологии $\Theta_{+}^{\text {loc }}($ см. замечание 2.1$)$ и поэтому она содержит сходящуюся подпоследовательность $\left\{\left(y_{m^{\prime}}(\cdot), z_{m^{\prime}}(\cdot)\right)\right\}:$ при $m^{\prime} \rightarrow \infty$

$$
\left(y_{m^{\prime}}(\cdot), z_{m^{\prime}}(\cdot)\right) \rightarrow(y(\cdot), z(\cdot)) \text { в } \Theta_{+}^{\text {loc }}
$$

для некоторой пары функций $(y(\cdot), z(\cdot)) \in \mathscr{F}_{+}^{\mathrm{b}}$. Это означает, что при любом $M>0$ при $m \rightarrow \infty$ имеют место следующие сходимости:

$$
\begin{aligned}
& \left.\begin{array}{l}
y_{m}(\cdot) \rightarrow y(\cdot) \quad * \text {-слабо в } L_{\infty}(0, M ; H) \\
y_{m}(\cdot) \rightarrow y(\cdot) \quad \text { слабо в } L_{2}(0, M ; V) \\
z_{m}(\cdot) \rightarrow z(\cdot) \quad * \text {-слабо в } L_{\infty}(0, M ; V)
\end{array}\right\}, \\
& \left.y_{m}(\cdot) \rightarrow y(\cdot) \quad \text { слабо в } L_{p_{1}}\left(0, M ; L_{p_{1}}(\Omega)\right)\right\} \\
& \left.z_{m}(\cdot) \rightarrow z(\cdot) \quad \text { слабо в } L_{p_{2}}\left(0, M ; L_{p_{2}}(\Omega)\right)\right\} \text {, } \\
& \left.\begin{array}{l}
\partial_{t} y_{m}(\cdot) \rightarrow \partial_{t} y(\cdot) \quad \text { слабо в } L_{q_{1}}\left(0, M ; H^{-r_{1}}(\Omega)\right) \\
\partial_{t} z_{m}(\cdot) \rightarrow \partial_{t} z(\cdot) \quad \text { слабо в } L_{q_{2}}\left(0, M ; H^{-r_{2}}(\Omega)\right)
\end{array}\right\} .
\end{aligned}
$$

Здесь мы для краткости обозначили индексы $m^{\prime}$ через $m$.

Зафиксируем произвольное число $M>0$ и воспользуемся рассуждениями из доказательства утверждения 2.2. Переходя, если необходимо, к подпоследовательности $\left\{m^{\prime}\right\}$, которую вновь обозначим через $\{m\}$, получим, что при $m \rightarrow \infty$

$$
\begin{aligned}
& f\left(y_{m}, z_{m}\right) \rightarrow f(y, z) \quad \text { слабо в } L_{q_{1}}\left(0, M ; L_{q_{1}}(\Omega)\right), \\
& h\left(y_{m}, z_{m}\right) \rightarrow h(y, z) \quad \text { слабо в } L_{q_{2}}\left(0, M ; L_{q_{2}}(\Omega)\right) .
\end{aligned}
$$

Из формулы во второй строке (4.6) следует, что при $m \rightarrow \infty$

$$
\Delta y_{m}(\cdot) \rightarrow \Delta y(\cdot) \quad \text { слабо в } L_{2}\left(0, M ; H^{-1}\right) .
$$

Напомним, что по условию последовательность $\left\{z_{m}(\cdot)\right\}$ ограничена в пространстве $L_{\infty}\left(\mathbb{R}_{+} ; V\right)$. Поэтому

$$
\left\|\delta_{m} \Delta z_{m}\right\|_{L_{\infty}\left(0, M ; H^{-1}\right)} \leqslant \delta_{m} C^{\prime}\left\|z_{m}\right\|_{L_{\infty}(0, M ; V)} \leqslant \delta_{m} C^{\prime} K \rightarrow 0, \quad \delta_{m} \rightarrow 0^{+},
$$

т.е. при $m \rightarrow \infty$

$$
\delta_{m} \Delta z_{m}(\cdot) \rightarrow 0 \quad \text { сильно в } L_{\infty}\left(0, M ; H^{-1}\right) .
$$

Доказанные выше сходимости (4.6)-(4.11) сильнее сходимости в пространстве обобщенных функций $\mathscr{D}^{\prime}\left(0, M ; H^{-r_{1}}(\Omega) \times H^{-r_{2}}(\Omega)\right)$. Совершая эти предельные 
переходы в системе (4.4)-(4.5), получаем, что функции $(y(t), z(t)), t \in] 0, M[$, удовлетворяют уравнениям

$$
\begin{aligned}
& \partial_{t} y=\Delta y-f(y, z)+g_{1}(x), \\
& \partial_{t} z=-h(y, z)+g_{2}(x)
\end{aligned}
$$

при любом $M>0$, т.е. $(y(t), z(t)), t \in \mathbb{R}_{+}$, является слабым решением системы (3.1)-(3.2). Осталось проверить (4.3). По условию $\left(y_{m}, z_{m}\right) \in \mathscr{K}_{+}^{\delta_{m}}(0)$, т.е. функции $z_{m}(\cdot)$ удовлетворяют неравенству

$$
\sup \left\{\left\|z_{m}(t)\right\|_{1}^{2} \mid t \geqslant 0\right\} \leqslant R^{2}, \quad m \in \mathbb{N} .
$$

Напомним, что

$$
z_{m}(\cdot) \rightarrow z(\cdot) * \text {-слабо в } L_{\infty}(0, M ; V), \quad M>0,
$$

поэтому

$$
\operatorname{ess} \sup \left\{\|z(t)\|_{1}^{2} \mid t \geqslant 0\right\} \leqslant \underline{\lim _{m \rightarrow \infty}} \operatorname{ess} \sup \left\{\left\|z_{m}(t)\right\|_{1}^{2} \mid t \geqslant 0\right\} \leqslant R^{2} .
$$

Напомним, что функция $\|z(t)\|_{1}^{2}$ полунепрерывна снизу при всех $t \geqslant 0$ и, следовательно,

$$
\sup \left\{\|z(t)\|_{1}^{2} \mid t \geqslant 0\right\} \leqslant R^{2} .
$$

Неравенство (3.13) установлено для функции $z(t)$ при $N=0$, т.е. $(y, z) \in \mathscr{K}_{+}^{0}(0)$.

Докажем теперь основную теорему о сходимости траекторных аттракторов.

Напомним, что траекторные аттракторы $\mathfrak{A}^{\delta}, 0<\delta \leqslant 1$, и $\mathfrak{A}^{0}$ ограничены в пространстве $\mathscr{F}_{+}^{\mathrm{b}}$ (см. следствие 2.1). Поэтому все они лежат внутри достаточно большого шара, т.е. $\mathscr{B}_{r} \subset \mathscr{F}_{+}^{\mathrm{b}}$ :

$$
\mathfrak{A}^{\delta} \subset \mathscr{B}_{r}, \quad 0 \leqslant \delta \leqslant 1 .
$$

Напомним, что топология $\Theta_{+}^{\text {loc }}$ метризуема на $\mathscr{B}_{r}$ (см. замечание 2.1).

Tеорема 4.1. Траекторные аттракторы $\mathfrak{A}^{\delta}$ системы (1.1)-(1.2) сходятся при $\delta \rightarrow 0^{+}$в топологии $\Theta_{+}^{\text {loc }} \kappa$ траекторному аттрактору $\mathfrak{A}^{0}$ системь $(3.1)-(3.2)$, m.e. $в \Theta_{+}^{\text {loc }}$

$$
\mathfrak{A}^{\delta} \rightarrow \mathfrak{A}^{0}, \quad \delta \rightarrow 0^{+} .
$$

ДокАзАтельство. Требуется установить, что для произвольной $\varepsilon$-окрестности $\mathscr{O}_{\varepsilon}\left(\mathfrak{A}^{0}\right)$ множества $\mathfrak{A}^{0}$ в топологии $\Theta_{+}^{\text {loc }}$ найдется число $\delta_{0}=\delta_{0}(\varepsilon)$ такое, что

$$
\mathfrak{A}^{\delta} \subset \mathscr{O}_{\varepsilon}\left(\mathfrak{A}^{0}\right), \quad \delta \leqslant \delta_{0},
$$

или, что эквивалентно,

$$
\mathfrak{A}^{\delta} \subset \mathscr{O}_{\varepsilon}\left(\Pi_{+} \mathscr{K}^{0}\right), \quad \delta \leqslant \delta_{0},
$$

где $\mathscr{K}^{0}$ - ядро системы (3.1)-(3.2) (см. равенство (3.17)). Предположим, что (4.17) неверно. Тогда найдется последовательность

$$
\delta_{m} \rightarrow 0^{+}, \quad m \rightarrow \infty,
$$


такая, что для некоторого $\varepsilon>0$

$$
\mathfrak{A}^{\delta_{m}} \not \subset \mathscr{O}_{\varepsilon}\left(\Pi_{+} \mathscr{K}^{0}\right) .
$$

Выберем произвольную последовательность $\tau_{m}>0$ такую, что

$$
\tau_{m} \rightarrow \infty, \quad m \rightarrow \infty
$$

Заметим, что

$$
T\left(\tau_{m}\right) \mathfrak{A}^{\delta_{m}}=\mathfrak{A}^{\delta_{m}}, \quad m \in \mathbb{N}
$$

(свойство строгой инвариантности траекторных аттракторов (2.20)). Следовательно, из (4.18) и (4.19) имеем

$$
T\left(\tau_{m}\right) \mathfrak{A}^{\delta_{m}} \not \subset \mathscr{O}_{\varepsilon}\left(\Pi_{+} \mathscr{K}^{0}\right) .
$$

Поэтому найдутся такие пары функций

$$
w_{m}(\cdot)=\left(u_{m}(\cdot), v_{m}(\cdot)\right) \in \mathfrak{A}^{\delta_{m}},
$$

для которых функции

$$
W_{m}(t):=T\left(\tau_{m}\right) w_{m}(t)=\left(u_{m}\left(t+\tau_{m}\right), v_{m}\left(t+\tau_{m}\right)\right), \quad t \geqslant 0,
$$

не принадлежат $\mathscr{O}_{\varepsilon}\left(\Pi_{+} \mathscr{K}^{0}\right)$, т.е.

$$
W_{m}(\cdot) \notin \mathscr{O}_{\varepsilon}\left(\Pi_{+} \mathscr{K}^{0}\right), \quad m \in \mathbb{N} .
$$

Также заметим, что пара $W_{m}(t)=\left(U_{m}(t), V_{m}(t)\right)$ является решением системы (1.1)-(1.2) с коэффициентом $\delta=\delta_{m}$ при $t \geqslant-\tau_{m}$, так как $\left(u_{m}\left(t+\tau_{m}\right), v_{m}\left(t+\tau_{m}\right)\right)$ является решением этой системы при $t+\tau_{m} \geqslant 0$, а система является автономной. Кроме того, в силу (4.21) выполнено неравенство

$$
\sup \left\{\left\|V_{m}(t)\right\|_{1} \mid t \geqslant-\tau_{m}\right\} \leqslant R,
$$

так как $\mathfrak{A}^{\delta_{m}} \subset \mathscr{K}^{\delta_{m}}(0)$ (см. утверждение 2.3).

Обозначим через $\mathscr{F}_{\ell}^{\text {loc }}$ и $\mathscr{F}_{\ell}^{\mathrm{b}}$ при $\ell \in \mathbb{R}$ пространства, аналогичные $\mathscr{F}_{0}^{\text {loc }}:=$ $\mathscr{F}_{+}^{\text {loc }}$ и $\mathscr{F}_{0}^{\mathrm{b}}:=\mathscr{F}_{+}^{\mathrm{b}}$, состоящие из функций, заданных на полупрямой $] \ell,+\infty[$ (см. (2.1)), а норма в $\mathscr{F}_{\ell}^{\mathrm{b}}$ определяется по формуле $(2.2)$ с заменой полупрямой $\left.\mathbb{R}_{+}=\right] 0,+\infty[$ на $] \ell,+\infty\left[\right.$. Аналогично $\Theta_{+}^{\text {loc }}$, в пространствах $\mathscr{F}_{\ell}^{\text {loc }}$ и $\mathscr{F}_{\ell}^{\mathrm{b}}$ определяется топология $\Theta_{\ell}^{\text {loc }}$.

Из (4.14) заключаем, что $W_{m}(t), t \geqslant-\tau_{m}$, принадлежит шару радиуса $r$ в пространстве $\mathscr{F}_{-\tau_{m}}^{\mathrm{b}}$, т.е.

$$
\left\|W_{m}(\cdot)\right\|_{\mathscr{F}_{-\tau_{m}}^{\mathrm{b}}} \leqslant r, \quad m \in \mathbb{N} .
$$

В силу замечания 2.1 при любом фиксированном $M>0$ последовательность $\left\{W_{m}(t), \tau_{m} \geqslant M\right\}$ является предкомпактной в пространстве $\Theta_{-M}^{\text {loc }}$. Значит, для любого $M>0$ имеется подпоследовательность $m^{\prime}=m^{\prime}(M)$, для которой $\left\{W_{m^{\prime}}(\cdot)\right\}$ - сходящаяся последовательностью в топологии $\Theta_{-M}^{\text {loc }}$. Тогда, используя известное диагональное построение Кантора, можно найти такую 
подпоследовательность индексов $\left\{m^{\prime \prime}\right\} \subset\{m\}$ и такую функцию $W(t), t \in \mathbb{R}$, что при $m^{\prime \prime} \rightarrow \infty$

$$
W_{m^{\prime \prime}}(\cdot) \rightarrow W(\cdot) \quad \text { в } \Theta_{-\infty}^{\text {loc }}=\Theta^{\text {loc }} .
$$

При этом в силу (4.24)

$$
\|W(\cdot)\|_{\mathscr{F} \mathrm{b}} \leqslant r
$$

т.е. $W \in \mathscr{F}^{\mathrm{b}}$, а в силу $(4.23)$

$$
\sup \left\{\|V(t)\|_{1} \mid t \in \mathbb{R}\right\} \leqslant R .
$$

Покажем, что функция $W(t), t \in \mathbb{R}$, принадлежит ядру $\mathscr{K}^{0}$ предельного уравнения (3.1)-(3.2). Действительно, применим к каждой сходящейся в $\Theta_{-M}^{\text {loc }}$ подпоследовательности $\left\{W_{m^{\prime}}(\cdot)\right\}$ утверждение 4.1 , в котором, очевидно, можно заменить полупрямую $[0,+\infty[$ на полупрямую $[-M,+\infty[$, так как рассматриваемая система реакции-диффузии является автономной. Следовательно, функция $W(t), t \geqslant-M$, является слабым решением системы (3.1)-(3.2) при любом $M>0$, а в силу (4.26) и (4.27) имеем

$$
W(\cdot) \in \mathscr{K}^{0} .
$$

Из (4.25) также заключаем, что

$$
\Pi_{+} W_{m^{\prime \prime}}(\cdot) \rightarrow \Pi_{+} W(\cdot), \quad m^{\prime \prime} \rightarrow \infty \quad \text { в } \quad \Theta_{0}^{\text {loc }} .
$$

Следовательно, если $m^{\prime \prime}$ достаточно велико, то

$$
\Pi_{+} W_{m^{\prime \prime}}(\cdot) \in \mathscr{O}_{\varepsilon}\left(\Pi_{+} W\right) \subset \mathscr{O}_{\varepsilon}\left(\Pi_{+} \mathscr{K}^{0}\right),
$$

что противоречит (4.22). Таким образом, свойство (4.15) выполнено.

Мы доказали также и

СЛЕДСТВИЕ 4.1. Ядра $\mathscr{K}^{\delta}$ системы (1.1)-(1.2) сходятся при $\delta \rightarrow 0^{+}$в топологии $\Theta^{\text {loc }}$ к ядру $\mathscr{K}^{0}$ системъ (3.1)-(3.2), m.e.

$$
\mathscr{K}^{\delta} \rightarrow \mathscr{K}^{0}\left(\delta \rightarrow 0^{+}\right) \quad \text { в } \Theta^{\text {loc }} .
$$

\section{Список литературы}

[1] R. Temam, Infinite-dimensional dynamical systems in mechanics and physics, Appl. Math. Sci., 68, Springer-Verlag, New York, 1988.

[2] А.В. Бабин, М. И. Вишик, Аттракторы эволюционных уравнений, Наука, М., 1989; англ. пер.: A. V. Babin, M. I. Vishik, Attractors of evolution equations, Stud. Math. Appl., 25, North-Holland, Amsterdam, 1992.

[3] V.V. Chepyzhov, M. I. Vishik, Attractors for equations of mathematical physics, Amer. Math. Soc. Colloq. Publ., 49, Amer. Math. Soc., Providence, RI, 2002.

[4] J. K. Hale, Asymptotic behavior of dissipative systems, Math. Surveys Monogr., 25, Amer. Math. Soc., Providence, RI, 1988.

[5] J. C. Robinson, Infinite-dimensional dynamical systems, An introduction to dissipative parabolic PDEs and the theory of global attractors, Cambridge Texts Appl. Math., Cambridge Univ. Press, Cambridge, 2001. 
[6] V. V. Chepyzhov, M. I. Vishik, "Trajectory attractors for evolution equations", C. $R$. Acad. Sci. Paris Sér. I Math., 321:10 (1995), 1309-1314.

[7] V. V. Chepyzhov, M. I. Vishik, "Trajectory attractors for reaction-diffusion systems", Topol. Methods Nonlinear Anal., 7:1 (1996), 49-76.

[8] V. V. Chepyzhov, M. I. Vishik, "Evolution equations and their trajectory attractors", J. Math. Pures Appl. (9), 76:10 (1997), 913-964.

[9] М.И. Вишик, В.В. Чепыжов, "Траекторный и глобальный аттракторы 3D системы Навье-Стокса", Матем. заметки, 71:2 (2002), 194-213; англ. пер.: M. I. Vishik, V. V. Chepyzhov, "Trajectory and global attractors of three-dimensional Navier-Stokes systems", Math. Notes, 71:1-2 (2002), 177-193.

[10] М.И. Вишик, В.В. Чепыжов, "Неавтономное уравнение Гинзбурга-Ландау и его аттракторы", Матем. сб., 196:6 (2005), 17-42; англ. пер.: M. I. Vishik, V.V. Chepyzhov, "Non-autonomous Ginzburg-Landau equation and its attractors", Sb. Math., 196:6 (2005), 791-815.

[11] M. Marion, "Finite-dimensional attractors associated with partly dissipative reaction-diffusion systems", SIAM J. Math. Anal., 20:4 (1989), 816-844.

[12] R. Fitz-Hugh, "Impulses and physiological states in theoretical models of nerve membranes", Biophys. J., 1:445-446 (1961).

[13] J. Nagumo, S. Arimoto, S. Yoshizawa, "An active pulse transmission line simulating nerve axon", Proc. IRE, 50:10 (1962), 2061-2070.

[14] Ж.-Л. Лионс, Некоторые методы решения нелинейных краевых задач, Мир, М., 1972; пер. с фр. J.-L. Lions, Quelques méthodes de résolution des problèmes aux limites non linéaires, Dunod, de Gruyter, Paris, 1969.

[15] Ж.-Л. Лионс, Э. Мадженес, Неоднородные граничные задачи и их приложения, т. I, Мир, М., 1971; пер. с фр.: J.-L. Lions, E. Magenes, Problèmes aux limites non homogènes et applications, vol. 1, Dunod, Paris, 1968.

[16] П. С. Александров, Введение в теорию множеств и теорию функиий. Ч. 1. Введение в общую теорию множеств и функиий, Гостехиздат, М., 1948.

[17] J.-P. Aubin, "Un théorème de compacite", C. R. Acad. Sci. Paris, 256 (1963), 5042-5044.

[18] Ю.А. Дубинский, "Слабая сходимость в нелинейных эллиптических и параболических уравнениях", Матем. сб., 67(109):4 (1965), 609-642.

М. И. Вишик (M. Io. Vishik)

Институт проблем передачи информации

им. А. А. Харкевича РАН

E-mail: vishik@iitp.ru

\section{В. В. Чепыжов (V. V. Chepyzhov)}

Институт проблем передачи информации

им. А. А. Харкевича РАН

E-mail: chep@iitp.ru
Поступила в редакцию

09.09.2008 